\title{
On Detectable and Non-detectable Structural Change
}

\author{
David F. Hendry* \\ Nuffield College, Oxford.
}

September 5, 1999

\begin{abstract}
A range of parameter changes in I(1) cointegrated time series are not reflected in econometric models thereof: many shifts are not easily detected by conventional tests. The breaks in question are changes that leave the unconditional expectations of the $\mathrm{I}(0)$ components unaltered. Thus, dynamics, adjustment speeds etc. may alter without detection. However, shifts in long-run means are generally noticeable. Using a VECM model class, we explain why this occurs, and illustrate the contrasting ease of detection of 'deterministic' and 'stochastic' shifts by Monte Carlo. The stark implications for 'impulse response analyses' are discussed.
\end{abstract}

\section{Introduction}

Structural change and dynamics are inherent facets of economic life, and over recorded history have revolutionized the human condition: statistical tests are not needed to discriminate between conditions in 1899 and 1999. Nevertheless, it transpires that a range of parameter changes in econometric models cannot be easily detected by conventional tests, whereas other changes are manifest and easy to detect. The former class includes changes that leave unaltered the unconditional expectations of non-integrated (denoted I(0)) components, even if dynamics, adjustment speeds, and intercepts are radically altered. The latter class comprises shifts in those unconditional expectations. Some related illustrations are provided in Hendry and Doornik (1997) and Clements and Hendry (1998).

This paper extends those studies, using a cointegrated vector autoregression (CVAR) as the general model. The analytical framework is the taxonomy of possible sources of forecast error developed in Clements and Hendry (1994), which highlights the impact of various forms of structural break in closed dynamic systems, and allows some surprising predictions about which parameters of a VAR can be altered without much impact on the properties of the resulting data. In matching Monte Carlo experiments, a bivariate system is subject to two structural breaks in its parameters, where the second re-instates the original parameter values, to show the contrast between the 'detectable' and (relatively) 'non-detectable' changes. ${ }^{1}$ For example, even when the generated data are integrated of order unity (I(1)), the intercepts and the dynamic coefficients of a CVAR can be changed considerably (i.e., by more than 50\%), yet parameter-constancy tests not detect such changes. Moreover, this is not due to model mis-specification: the in-sample model considered here coincides with the data generation process (DGP). Nor is it a matter of generically low-powered tests: the same tests have high power to detect

\footnotetext{
*Financial support from the U.K. Economic and Social Research Council under grant R000237500 is gratefully acknowledged. I am pleased to acknowledge helpful comments from Mike Clements, Jurgen Doornik, Bronwyn Hall, Grayham Mizon, John Muellbauer and Bent Nielsen.

${ }^{1}$ Of course, for a sufficiently-large sample, both pre and post any change, and for a big enough break, all shifts are detectable on the tests used here. The practical issue, however, is their ease of detection for realistic sample sizes and breaks of a plausible magnitude.
} 
other shifts in either intercepts or dynamic coefficients. The paper explains why this outcome occurs: for more general studies of the size and power properties of parameter-constancy tests in both $\mathrm{I}(0)$ and I(1) systems, see Anderson, Mizon and O’Brien (1993) and Hylleberg, Mizon and O’Brien (1993).

To summarize why non-detectability occurs, let $\mathbf{y}_{t}$ denote a vector $\mathrm{I}(0)$ time series with actual unconditional expectations and variances denoted by $\mathrm{E}\left[\mathrm{y}_{t}\right]$ and $\mathrm{V}\left[\mathrm{y}_{t}\right]$ respectively. Let the corresponding expectations based on assuming the model is the in-sample DGP be denoted by $\mathcal{E}\left[\mathbf{y}_{t}\right]$ and $\mathcal{V}\left[\mathbf{y}_{t}\right]$ (an alternative notation might be $\left.\mathrm{E}_{\mathrm{M}}[\cdot]\right)$. Then, so long as $\mathcal{V}\left[\mathbf{y}_{t}\right]$ is not markedly different from $\mathrm{V}\left[\mathrm{y}_{t}\right]$, detectability depends strongly on the difference $\mathrm{E}\left[\mathbf{y}_{t}\right]-\mathcal{E}\left[\mathbf{y}_{t}\right]$. Consequently, parameter changes in the class that leave $\mathrm{E}\left[\mathbf{y}_{t}\right] \simeq \mathcal{E}\left[\mathbf{y}_{t}\right]$ suffer from a detectability problem unless they generate large variance increases. Since I(1) CVARs can be reparameterized by differencing and cointegration transformations as $\mathrm{I}(0)$ vector equilibrium-correction models (VEqCMs) where all variables are expressed as deviations around their (pre-break) means, the same logic applies: only shifts in those means induce departures that are readily detectable. Indeed, it is easy to create major parameter shifts that leave the first two moments virtually unchanged, and such breaks will be almost impossible to detect using tests based on those moments. Although other aspects of the model might reveal such shifts, including residual autocorrelation when dynamics alter, the correct interpretation would not be obvious, and indeed other tests could well be distorted by the mis-specification deriving from the break. Different tests might be developed to prise out the correct underlying state of nature, but here we focus on the properties of recursively-computed F-tests of parameter constancy, and the associated recursive parameter estimates.

The structure of the paper is as follows. Section 2 describes the $n$-dimensional I(1) DGP that forms the class within which the analysis is undertaken. Section 3 formalizes the structural breaks to be studied, and $\S 3.1$ discusses which changes are and are not easily detected. This is followed by a Monte Carlo study of a bivariate I(1) CVAR in $\S 4$, for three classes of structural break, and in $\S 4.5$ for shifts in the cointegration space. Section 5 offers potential explanations for the mixture of results obtained and $\S 7$ concludes.

\section{The data generation process}

Consider a first-order vector autoregression in $n$ variables denoted $\mathbf{x}_{t}$ :

$$
\mathbf{x}_{t}=\boldsymbol{\tau}+\boldsymbol{\Gamma} \mathbf{x}_{t-1}+\nu_{t}
$$

where $\boldsymbol{\nu}_{t} \sim \mathrm{IN}_{n}\left[\mathbf{0}, \boldsymbol{\Omega}_{\nu}\right]$ denotes an independent, normal error with expectation $\mathrm{E}\left[\boldsymbol{\nu}_{t}\right]=\mathbf{0}$ and variance matrix $\mathrm{V}\left[\boldsymbol{\nu}_{t}\right]=\boldsymbol{\Omega}_{\nu}$. The data are assumed to be integrated of order unity (I (1)), perhaps after suitable transformations (such as logs), represented by:

$$
\boldsymbol{\Gamma}=\mathbf{I}_{n}+\boldsymbol{\alpha} \boldsymbol{\beta}^{\prime}
$$

where $\boldsymbol{\alpha}$ and $\boldsymbol{\beta}$ are $n \times r$ matrices of rank $r$. All eigenvalues of $\boldsymbol{\Gamma}$ lie on or inside the unit circle, to exclude explosive processes, and to exclude I $(2), \operatorname{rank}\left(\boldsymbol{\alpha}_{\perp}^{\prime} \boldsymbol{\Gamma} \boldsymbol{\beta}_{\perp}\right)=(n-r)$, where $\boldsymbol{\alpha}_{\perp}$ and $\boldsymbol{\beta}_{\perp}$ are $n \times(n-r)$ matrices of rank $(n-r)$ and $\boldsymbol{\alpha}^{\prime} \boldsymbol{\alpha}_{\perp}=\boldsymbol{\beta}^{\prime} \boldsymbol{\beta}_{\perp}=\mathbf{0}$ (although the analysis could be generalized to accommodate I(2) data). Additional lags are easily added, but do not seem to materially affect the outcomes, although linearity may do (primarily because most non-linear functions entail nonzero means, an issue of importance below). The parameters will be allowed to shift, which induces structural change in the system.

In (1):

$$
\tau=\gamma-\alpha \mu
$$


where $\boldsymbol{\mu}$ is $r \times 1$, and to ensure the appropriate number of 'free' parameters, $\boldsymbol{\beta}^{\prime} \boldsymbol{\gamma}=\mathbf{0}$. Substituting (2) and (3) into (1) yields the vector equilibrium-correction model (denoted VEqCM):

$$
\Delta \mathbf{x}_{t}-\gamma=\boldsymbol{\alpha}\left(\boldsymbol{\beta}^{\prime} \mathbf{x}_{t-1}-\boldsymbol{\mu}\right)+\boldsymbol{\nu}_{t}
$$

where $\Delta \mathbf{x}_{t}$ and $\boldsymbol{\beta}^{\prime} \mathbf{x}_{t}$ are I(0). From (4), $\mathrm{E}\left[\Delta \mathbf{x}_{t}\right]=\boldsymbol{\gamma}$ (the unconditional growth rate) so $\mathrm{E}\left[\boldsymbol{\beta}^{\prime} \mathbf{x}_{t}\right]=\boldsymbol{\mu}$ defines the equilibrium means.

\subsection{Parameter-constancy tests}

The statistics reported below for testing parameter constancy are recursively-computed, system variants of Chow (1960) tests, as described in Doornik and Hendry (1997, Ch. 14). In a univariate setting, the tests are calculated from the residual sums of squares as the sample size is increased, and so are related to the CUSUMSQ statistic in Brown, Durbin and Evans (1975). The vector F-tests use the F-approximation in Rao (1952), as described in Rao (1973, Section 8c.5) or Anderson (1984, Section 8.5.4): for other approaches, see (inter alia) Anderson and Mizon (1983).

\section{Structural change}

Under structural change, any or all of $\gamma, \boldsymbol{\alpha}, \boldsymbol{\beta}, \boldsymbol{\mu}$, or $\boldsymbol{\Omega}_{\nu}$ could alter (or indeed the forms of the relationships in the system, the distributional assumptions, and lag lengths, but these are not considered here). Situations such as economic transition undoubtedly involve changes to cointegration links and growth rates, as well as to speeds of adjustment and equilibrium means. We will focus on the more 'normal' setting where $\gamma, \boldsymbol{\alpha}$, or $\boldsymbol{\mu}$ shift. However, changes that involve setting $\boldsymbol{\alpha}=\mathbf{0}$, or changing $\boldsymbol{\alpha}=\mathbf{0}$ to a non-zero value, inherently involve changes in the cointegration structure, and these are investigated below. Changes to $\boldsymbol{\beta}$ could be studied, but pose rather different problems, as discussed later. Finally, changes to $\Omega_{\nu}$ are predicted by the following theory to have less of an impact, unless they are very large, so are not considered here.

Individual realizations of integrated time series almost inherently have non-zero data means, by force of their stochastic trends. Nevertheless, the $\mathrm{I}(0)$ components could have zero unconditional means, depending on the form of data transformation and units of measurement. Thus, in order to clarify the impacts of parameter changes, we also write the system (4) in I(0) space for the $n$ variables $\mathbf{y}_{t}$, the first $r$ of which are the $\boldsymbol{\beta}^{\prime} \mathbf{x}_{t}$ and the remaining $n-r$ are the relevant elements of $\Delta \mathbf{x}_{t}$ (e.g., $\boldsymbol{\alpha}_{\perp}^{\prime} \Delta \mathbf{x}_{t}$ which ensures a non-singular representation). Of course, this representation is inappropriate when $\boldsymbol{\beta}$ can alter. Thus:

$$
\mathbf{y}_{t}=\phi+\boldsymbol{\Pi}_{t-1}+\epsilon_{t} \text { with } \boldsymbol{\epsilon}_{t} \sim \mathrm{IN}_{n}\left[\mathbf{0}, \boldsymbol{\Omega}_{\epsilon}\right] .
$$

The unconditional expectation (or long-run mean) $\mathrm{E}\left[\mathbf{y}_{t}\right]$ of $\mathbf{y}_{t}$ over $t=1, \ldots, T$ is:

$$
\mathrm{E}\left[\mathbf{y}_{t}\right]=\left(\mathbf{I}_{n}-\mathbf{\Pi}\right)^{-1} \phi=\varphi
$$

since the earlier assumptions ensure that $\Pi$ has all its eigenvalues less than unity in absolute value, leading to the homogeneous specification:

$$
\mathbf{y}_{t}-\varphi=\Pi\left(\mathbf{y}_{t-1}-\varphi\right)+\epsilon_{t}
$$

Under the assumption of constant parameters, (7) would deliver the 1-step ahead outcome (assuming known parameters):

$$
\mathbf{y}_{T+1}-\boldsymbol{\varphi}=\boldsymbol{\Pi}\left(\mathbf{y}_{T}-\boldsymbol{\varphi}\right)+\boldsymbol{\epsilon}_{T+1} .
$$


At time period $T$, however, we let $(\phi: \Pi)$ change to $\left(\phi^{*}: \Pi^{*}\right)$, so from $T+1$ onwards, the data are generated by:

$$
\mathbf{y}_{T+h}=\phi^{*}+\boldsymbol{\Pi}^{*} \mathbf{y}_{T+h-1}+\boldsymbol{\epsilon}_{T+h}, \quad h \geq 1 .
$$

We assume $\Pi^{*}$ still has all its eigenvalues less than unity in absolute value, and that the number and form of the cointegrating vectors remains the same. Let $\varphi^{*}=\left(\mathbf{I}_{n}-\mathbf{\Pi}^{*}\right)^{-1} \boldsymbol{\phi}^{*}$, then:

$$
\mathbf{y}_{T+h}-\varphi^{*}=\boldsymbol{\Pi}^{*}\left(\mathbf{y}_{T+h-1}-\varphi^{*}\right)+\boldsymbol{\epsilon}_{T+h} .
$$

The thrust of Hendry and Doornik (1997), building on Clements and Hendry (1994), is that changes in $\varphi$ are easy to detect, whereas those in $\phi$ and $\Pi$ are not when $\varphi$ is unchanged, so we consider that issue first. We refer to shifts in $\varphi$ as 'deterministic' changes (since they change the long-run mean), whereas shifts in $\phi$ and $\Pi$ that leave $\varphi$ unchanged are referred to as 'stochastic' or 'non-deterministic', even though they might involve a changed intercept.

Let $\boldsymbol{\epsilon}_{T+1 \mid T}$ denote the difference between (8) and (9) at 1-step ahead:

$$
\begin{aligned}
\boldsymbol{\epsilon}_{T+1 \mid T} & =\boldsymbol{\varphi}^{*}+\boldsymbol{\Pi}^{*}\left(\mathbf{y}_{T}-\boldsymbol{\varphi}^{*}\right)+\boldsymbol{\epsilon}_{T+1}-\left[\boldsymbol{\varphi}+\boldsymbol{\Pi}\left(\mathbf{y}_{T}-\boldsymbol{\varphi}\right)+\boldsymbol{\epsilon}_{T+1}\right] \\
& =\boldsymbol{\varphi}^{*}-\boldsymbol{\varphi}+\boldsymbol{\Pi}^{*}\left(\mathbf{y}_{T}-\boldsymbol{\varphi}^{*}\right)-\boldsymbol{\Pi}\left(\mathbf{y}_{T}-\boldsymbol{\varphi}\right) \\
& =\left(\mathbf{I}_{n}-\boldsymbol{\Pi}^{*}\right)\left(\boldsymbol{\varphi}^{*}-\boldsymbol{\varphi}\right)+\left(\boldsymbol{\Pi}^{*}-\boldsymbol{\Pi}\right)\left(\mathbf{y}_{T}-\boldsymbol{\varphi}\right) .
\end{aligned}
$$

This would be the expected 1-step ahead forecast error from using (8) as a model when (9) is the DGP, and all parameters are known. The first term on the last line is the equilibrium-mean shift, and the second is the slope change, which has an unconditional expectation of zero. Indeed, if a slope change occurred when the economy was near equilibrium, so $\mathbf{y}_{T} \simeq \boldsymbol{\varphi}$ and $\boldsymbol{\varphi} \operatorname{did}$ not alter, then $\epsilon_{T+1 \mid T} \simeq \mathbf{0}$. Of course, if the economy was in substantial disequilibrium, a larger effect would result, but this seems to part of the explanation for the non-detectability of shifts in $\phi$ and $\Pi$ that leave $\varphi$ unchanged.

\subsection{Detectable shifts}

We now show that deterministic shifts, namely shifts from $\varphi$ to $\varphi^{*}$, whether induced by changes in the intercept $\phi$, or indirectly by changes in dynamics (i.e., via $\left(\mathbf{I}_{n}-\boldsymbol{\Pi}^{*}\right)^{-1} \phi$ ) are a detectable failure in linear dynamic econometric systems. We then demonstrate that even if every parameter alters, but this leaves the 'long-run means' $\varphi$ unchanged, so $\phi^{*}=\left(\mathbf{I}_{n}-\Pi^{*}\right) \varphi$, structural change is not easily detected. Indeed, shifts in $\phi, \Pi$ with constant $\varphi$ transpire to be isomorphic to changes in mean-zero processes, where $\phi=\phi^{*}=\mathbf{0}$. Further, while breaks in $\Pi$ alone can cause forecast failures, their ease of detection depends on the magnitudes of the long-run means of the $\mathrm{I}(0)$ components relative to their error standard deviations. As Hendry and Doornik (1997) remark, when the long-run mean is non-zero, breaks shift the location of the data, inducing a short-run 'trend' to the new equilibrium mean, which is more easily detected than a one-off variance change around the origin. If (4) had additional dynamics, these could be expressed in growth-rate form, and hence re-written to have zero means around (the equivalent of) $\gamma$, so the same argument about detectability would apply to shifts in their parameters.

Because the system is dynamic, the impact of any break takes time to have its full effect, and for the system to reach a new equilibrium, so the data expectations alter in every period, making the process highly non-stationary. To develop the primary implications, we just consider the first period following a change, denoted time $T$ : later periods are amenable to analysis, as are successive changes, although the algebra becomes increasingly complicated. Let ${ }^{\wedge}$ denote an estimate, so the forecast error at $T+1$, immediately after the break (or ex post residual using parameter estimates up to $T$ ), is $\hat{\boldsymbol{\epsilon}}_{T+1 \mid T}=$ $\mathbf{y}_{T+1}-\hat{\mathbf{y}}_{T+1 \mid T}$ where:

$$
\hat{\boldsymbol{\epsilon}}_{T+1 \mid T}=\boldsymbol{\phi}^{*}+\boldsymbol{\Pi}^{*} \mathbf{y}_{T}+\boldsymbol{\epsilon}_{T+1}-\hat{\boldsymbol{\phi}}-\hat{\boldsymbol{\Pi}} \mathbf{y}_{T}
$$


We treat finite-sample biases in estimators as negligible (see Hendry, 1997, for an explanation), so set $\mathrm{E}[\hat{\mathbf{\Pi}}]=\Pi$ and $\mathrm{E}[\hat{\boldsymbol{\phi}}]=\phi$. Further, as almost all estimation methods match data means in-sample, $\hat{\boldsymbol{\varphi}}=\left(\mathbf{I}_{n}-\hat{\mathbf{\Pi}}\right)^{-1} \hat{\boldsymbol{\phi}}$. Let $\mathcal{E}$ denote the expected value computed from the model, namely, the actual mean of the forecasts given the in-sample parameter values, when (5) is assumed to hold (i.e., in ignorance of the shift), then conditional on $\mathbf{y}_{T}$ :

$$
\mathcal{E}\left[\hat{\mathbf{y}}_{T+1 \mid T} \mid \mathbf{y}_{T}\right]=\boldsymbol{\phi}+\boldsymbol{\Pi} \mathbf{y}_{T} .
$$

This is to be contrasted with the actual data expectation at $T+1$ :

$$
\mathrm{E}\left[\mathbf{y}_{T+1} \mid \mathbf{y}_{T}\right]=\boldsymbol{\phi}^{*}+\mathbf{\Pi}^{*} \mathbf{y}_{T} .
$$

Then, detectability depends primarily on $\mathrm{E}\left[\mathbf{y}_{T+1} \mid \mathbf{y}_{T}\right]-\mathcal{E}\left[\hat{\mathbf{y}}_{T+1 \mid T} \mid \mathbf{y}_{T}\right]$. More precisely from (11):

$$
\begin{aligned}
\mathrm{E}\left[\hat{\boldsymbol{\epsilon}}_{T+1 \mid T} \mid \mathbf{y}_{T}\right] & =\boldsymbol{\phi}^{*}+\boldsymbol{\Pi}^{*} \mathbf{y}_{T}+\mathrm{E}\left[\boldsymbol{\epsilon}_{T+1}\right]-\mathrm{E}[\hat{\boldsymbol{\phi}}]-\mathrm{E}[\hat{\boldsymbol{\Pi}}] \mathbf{y}_{T} \\
& =\boldsymbol{\phi}^{*}-\boldsymbol{\phi}+\left(\boldsymbol{\Pi}^{*}-\mathbf{\Pi}\right) \mathbf{y}_{T} \\
& =\mathrm{E}\left[\mathbf{y}_{T+1} \mid \mathbf{y}_{T}\right]-\mathcal{E}\left[\hat{\mathbf{y}}_{T+1 \mid T} \mid \mathbf{y}_{T}\right] .
\end{aligned}
$$

Moreover, taking the unconditional means of each term using (6):

$$
\mathcal{E}\left[\hat{\mathbf{y}}_{T+1 \mid T}\right]=\phi+\Pi E\left[\mathbf{y}_{T}\right]=\left(\mathbf{I}_{n}-\boldsymbol{\Pi}\right) \boldsymbol{\varphi}+\boldsymbol{\Pi} \boldsymbol{\varphi}=\boldsymbol{\varphi}
$$

whereas:

$$
\begin{aligned}
\mathrm{E}\left[\mathbf{y}_{T+1}\right] & =\phi^{*}+\boldsymbol{\Pi}^{*} \boldsymbol{\varphi}=\left(\mathbf{I}_{n}-\boldsymbol{\Pi}^{*}\right) \varphi^{*}+\boldsymbol{\Pi}^{*} \varphi^{*}-\boldsymbol{\Pi}^{*}\left(\varphi^{*}-\varphi\right) \\
& =\varphi^{*}-\boldsymbol{\Pi}^{*}\left(\varphi^{*}-\varphi\right),
\end{aligned}
$$

so unconditionally:

$$
\mathrm{E}\left[\mathbf{y}_{T+1}\right]-\mathcal{E}\left[\hat{\mathbf{y}}_{T+1 \mid T}\right]=\mathrm{E}\left[\hat{\boldsymbol{\epsilon}}_{T+1}\right]=\left(\mathbf{I}_{n}-\mathbf{\Pi}^{*}\right)\left(\boldsymbol{\varphi}^{*}-\boldsymbol{\varphi}\right) .
$$

This is simply the first term in the last line of (10)

The key implication from (14) is that $\mathrm{E}\left[\mathbf{y}_{T+1}\right]=\mathcal{E}\left[\hat{\mathbf{y}}_{T+1 \mid T}\right]$ when $\varphi^{*}=\boldsymbol{\varphi}$. This can occur despite changes in the dynamics, represented by shifts in $\Pi$, or changes in $\phi$ : for example, if $\left\{\mathbf{y}_{t}\right\}$ is a meanzero process; if $\varphi$ does not change; or if shifts in $\phi^{*}$ offset those in $\Pi^{*}$ to leave $\varphi$ constant, despite both dynamics and intercepts shifting. Further, for a given value of $\left(\varphi^{*}-\varphi\right)$, the effect is larger or smaller as $\left(\mathbf{I}_{n}-\boldsymbol{\Pi}^{*}\right)$ moves closer to (further from) zero.

Of course, the detectability of any shifts depends on their magnitude relative to the error standard deviations. Let $\boldsymbol{\Omega}_{\epsilon}^{-1}=\mathbf{K}^{\prime} \mathbf{K}$ so that:

$$
\mathbf{K} \mathbf{y}_{t}=\mathbf{K} \boldsymbol{\phi}+\mathbf{K} \Pi \mathbf{K}^{-1} \mathbf{K} \mathbf{y}_{t-1}+\mathbf{K} \boldsymbol{\epsilon}_{t}
$$

or letting $\mathbf{y}_{t}^{+}=\mathbf{K} \mathbf{y}_{t}$ :

$$
\mathbf{y}_{t}^{+}=\boldsymbol{\psi}+\mathbf{\Psi y}_{t-1}^{+}+\boldsymbol{\epsilon}_{t}^{+}
$$

where $\mathbf{K} \phi, \boldsymbol{\epsilon}_{t}^{+}=\mathbf{K} \boldsymbol{\epsilon}_{t} \sim \operatorname{IN}_{n}\left[\mathbf{0}, \mathbf{I}_{n}\right]$ and $\boldsymbol{\Psi}=\mathbf{K} \boldsymbol{\Pi} \mathbf{K}^{-1}$ (since $\boldsymbol{\Psi}$ and $\boldsymbol{\Pi}$ are related by a similarity transform, the ordering-dependence of the transformation by $\mathbf{K}$ does not matter here). Then, after the break:

$$
\mathrm{E}\left[\mathbf{y}_{T+1}^{+}\right]-\mathcal{E}\left[\hat{\mathbf{y}}_{T+1 \mid T}^{+}\right]=\mathbf{K}\left(\mathbf{I}_{n}-\mathbf{\Pi}^{*}\right)\left(\boldsymbol{\varphi}^{*}-\boldsymbol{\varphi}\right) .
$$

This is the appropriate metric for judging the 'magnitude' of a break.

We now illustrate the implications of this analysis for detecting structural change, using some numerical simulations of parameter-constancy tests. Although the above analysis only applies to one-step ahead errors after a single break, the Monte Carlo will examine many forecast (break-test) horizons, and allows for two shifts. Nevertheless, the implications will be seen to hold more generally. 


\section{An I(1) Monte Carlo}

The Monte Carlo simulation considers the following experiments, implemented in Ox by PcNaive for Windows (see Doornik, 1996, and Doornik and Hendry, 1998). The data are generated by a bivariate cointegrated I(1) VAR:

$$
\begin{aligned}
& \Delta x_{1, t}=\gamma_{1}+\alpha_{1}\left(x_{1, t-1}-x_{2, t-1}-\mu_{1}\right)+\epsilon_{1, t} \\
& \Delta x_{2, t}=\gamma_{2}+\alpha_{2}\left(x_{1, t-1}-x_{2, t-1}-\mu_{1}\right)+\epsilon_{2, t}
\end{aligned}
$$

where $\epsilon_{i, t} \sim \mathbb{N}\left[0, \sigma_{i i}\right]$, with $\mathbf{E}\left[\epsilon_{1, t} \epsilon_{2, s}\right]=0 \forall t, s$, so in (15), $\mathbf{K}$ is diagonal with elements $\sqrt{ } \sigma_{i i}$. We consider 4 types of experiment:

(A) a constant DGP (to establish actual test size);

(B) breaks in the coefficients of the feedbacks $\left(\alpha_{1}\right.$ and $\left.\alpha_{2}\right)$;

(C) breaks in the long-run mean $\left(\mu_{1}\right)$;

(D) breaks in the growth rates $\left(\gamma_{1}\right.$ and $\left.\gamma_{2}\right)$.

Four different full-sample sizes are considered: $T=24,60,100$, and 200 (denoted a, b, c, d on graphs), the last of which is relatively large for macro-economic models. Breaks occur at $t=0.5 T$, and revert to the original parameter values at $t=0.75 T$ to mimic a second break. Thus, the design seeks to isolate the effects of more information at larger $T$ with fixed relative break points: as will be seen, 'undetectable' breaks remain hard to find even at the largest sample size considered here. Since breaks are involved, these experiments could not have been conducted as one recursive experiment from $T=10$ to $T=200$, except when studying null rejection frequencies. However, for graphical presentation, the individual graph lines between $\mathrm{a}, \mathrm{b}, \mathrm{c}, \mathrm{d}$ are only distinguished by a different symbol where that clarifies an important feature, even when the recorded outcomes overlap.

The unrestricted VAR with intercept and one lag is estimated and tested. When breaks occur, modelling cointegrated processes is difficult, and as a VAR is usually the first step, constancy tests should be implemented at that stage (i.e., prior to checking cointegration): detectability may increase if a VECM form is imposed, but doing so is unlikely to affect the rankings across the outcomes in our experiments A-D. Throughout, 500 replications were used, and rejection frequencies at both 0.05 and 0.01 nominal test sizes were recorded (so have standard errors of about 0.01 and 0.004 respectively).

The experimental formulation is invariant to what induces changes in $\gamma_{i}-\alpha_{i} \mu_{1}$, but large growthrate changes seem unlikely in real economic variables. It may seem surprising that the 'absolute' size of $\mu_{1}$ can matter, since even after log transforms, the measurement units affect $\mu_{1}$ : for example, a switch from a price index normalized at unity to one normalized at 100 radically alters $\mu_{1}$ in (say) a log-linear money-demand model without affecting either $\alpha$ or $\sigma$. Nevertheless, changes in $\mu_{1}$ (relative to error standard deviations) also need to be judged absolutely, not as percentages: thus, using $\nabla$ to denote parameter changes, $\nabla \mu_{1} / \sigma$ matters per se, and this cannot depend on the measurement system, only on agents' behaviour. When $\mu_{1} / \sigma$ is large (small), a given effect will be a small (large) percent, but will have the same detectability for a given $\alpha$. For example, for both broad and narrow money demand in the UK after financial innovation in the mid 1980s (see Ericsson, Hendry and Prestwich, 1998, and Hendry and Ericsson, 1991), $\nabla \mu_{1} / \sigma \simeq 25-30$, in models that excluded appropriate modifiers. The rise in the savings rate in the mid 1970s was of roughly the same absolute magnitude (see e.g., Hendry, 1994). For 'standard' values of $\alpha$, (around 0.1-0.2) these numbers translate into 'permanent' equilibrium shifts of $2.5 \sigma$ to $6 \sigma$. Such considerations determined the values of the parameters in the experimental design.

Two baseline sets of dynamics are considered: $\alpha_{1}=-0.1$, and $\alpha_{2}=0$ (so $x_{2, t}$ is both weakly and strongly exogenous for the parameters of the first equation: see Engle, Hendry and Richard, 1983); and 
$\alpha_{1}=-0.1$, and $\alpha_{2}=0.1$ (so $x_{2, t}$ is neither weakly nor strongly exogenous for the parameters of the first equation). For $\alpha_{1}$, the change is -0.05 . We investigate $\mu_{1}=1$, changed by an amount of +0.3 for its break (so $\nabla \alpha_{1} \mu_{1}=3 \sigma_{11}$ ). Also, using $\boldsymbol{\beta}^{\prime}=(1:-1)$ enforces $\gamma_{1}=\gamma_{2}$ which was set to 0.01 (roughly $4 \%$ p.a. for quarterly data): the change considered is to double both of these to 0.02 in (D), which would constitute a very dramatic increase in long-term growth. Thus, two ratios of $\gamma_{i} / \sqrt{ } \sigma_{i i}$ are examined, namely unity and 2 (see Hylleberg and Mizon, 1989), inducing the derived values of $\sqrt{ } \sigma_{i i}=0.01$ throughout (roughly $1 \%$ for the 1 -step ahead forecast standard error under constant parameters). Notice that $\gamma$ and $\mu$ correspond to elements of $\varphi$ rather than $\phi$. In total, there are 8 baseline experiments, and the same number of changes to $\alpha, \gamma$, and $\mu$ making 32 experiments in total. These are reported graphically, each panel showing all four sample size outcomes for both $p$ values. The critical values for the constancy tests are those for a known break point, which delivers the highest possible power for the test used. The graphs serve to illustrate the outcomes visually, showing that rejection frequencies are everywhere low in some cases, confirming that the highest power is immediately before the first break, whereas the second break is often less detectable when the first has not been modelled, and sometimes showing that the tests are actually biased after the second break.

\subsection{Test size}

The relation of the actual to the nominal size for vector constancy tests has not been much investigated, so the experiments in (A) check their size in an I(1), cointegrated setting, with and without feedback in the second equation. As fig. 1 reveals, the results are reasonably reassuring when the EqCM enters both relations: with 500 replications, the approximate $95 \%$ confidence intervals are $(0.03,0.07)$ and $(0.002,0.018)$ for $5 \%$ and $1 \%$ nominal, and these are shown on the graphs as dotted and dashed lines respectively, revealing that few null rejection frequencies lie outside those bounds once the sample size exceeds 60 . At the smallest sample sizes, there is some over-rejection, though never above $9 \%$ for the 5\% nominal or $3 \%$ for the $1 \%$ nominal. When the $\mathrm{EqCM}$ enters the first relation only, there is a systematic, but small, excess rejection: around $6 \%$ instead of 5\%, and $1.5 \%$ instead of $1 \%$. However, these outcomes are not sufficiently discrepant to markedly affect the outcomes of the 'power' comparisons below.

\subsection{Dynamic shift}

Experiments in (B) demonstrate that a change in the strength of reaction to a zero-mean disequilibrium is not readily detectable. This is despite the fact that the intercept also shifts in the VAR representation:

$$
\begin{aligned}
& \mathbf{x}_{t}=\boldsymbol{\gamma}-\boldsymbol{\alpha} \boldsymbol{\mu}+\boldsymbol{\alpha} \boldsymbol{\beta}^{\prime} \mathbf{x}_{t-1}+\boldsymbol{\epsilon}_{t} \text { pre break } \\
& \mathbf{x}_{s}=\boldsymbol{\gamma}-\boldsymbol{\alpha}^{*} \boldsymbol{\mu}+\boldsymbol{\alpha}^{*} \boldsymbol{\beta}^{\prime} \mathbf{x}_{s-1}+\boldsymbol{\epsilon}_{s} \text { post break. }
\end{aligned}
$$

One might have anticipated detectablity from $\nabla \boldsymbol{\alpha} \boldsymbol{\mu} \neq \mathbf{0}$, particularly since that change numerically exceeds the equivalent jump in (C) which we show below is easily detected. Nevertheless, despite the induced intercept shift, changes in the dynamics alone are not easily detectable. Figure 2 records the outcomes: the powers are so low, do not increase with sample size, and indeed barely reflect any breaks, that one might question whether the Monte Carlo was correctly implemented: be assured it was, but anyway, this effect is easy to replicate using PcNaive. Moreover, it was predicted by the analysis above, by that in Clements and Hendry (1994), and has been found in a different setting by Hendry and Doornik (1997), so is not likely to be spurious.

Further, the presence of an additional EqCM feedback does not influence these results, even though one might expect an induced shift. Perhaps other tests could detect this type of change, but they will need 
No shift under weak exogeneity of $x \_\{2, t\}$

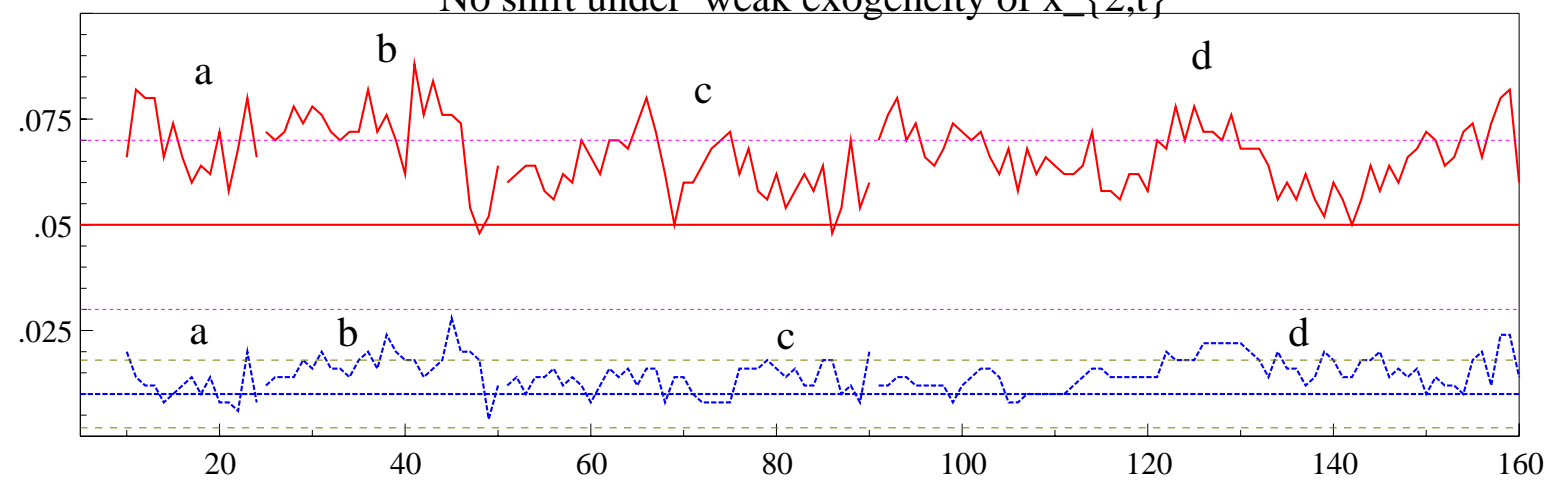

No shift and no weak exogeneity of $x_{-}\{2, t\}$

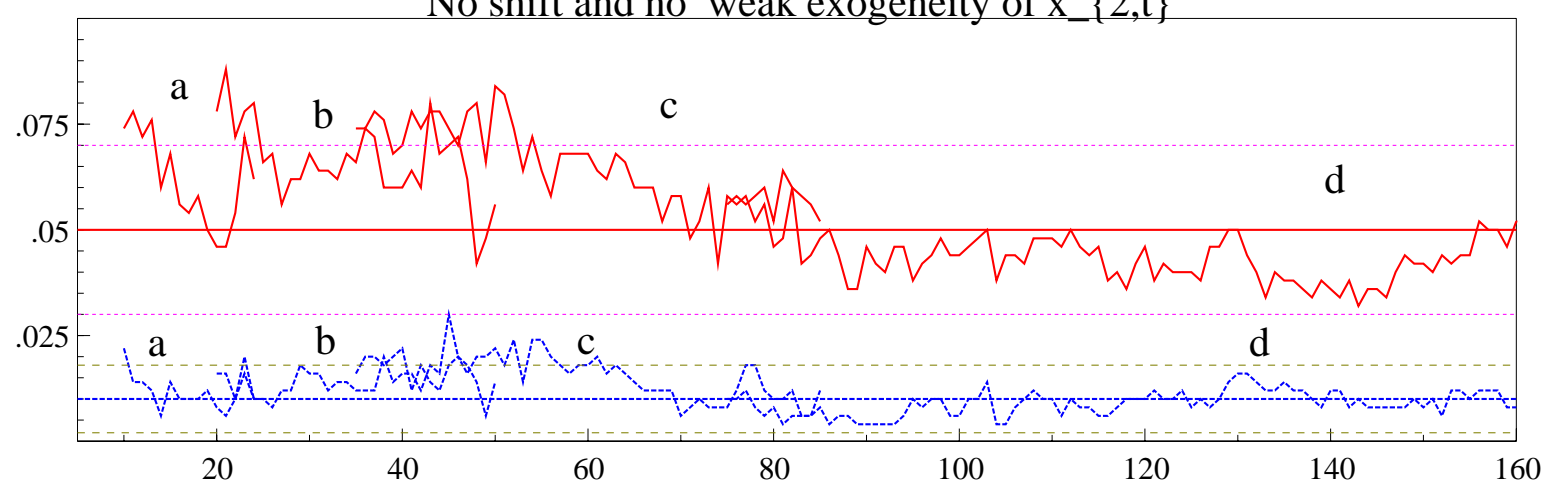

Figure 1 Constancy-test null rejection frequencies in a cointegrated process.

some other principle of construction than that used here if power is to be noticeably increased. Although direct testing of the parameters seems an obvious approach, as fig. 3 shows, there is no evidence in the recursive graphs of any marked change in estimates for the experiments where $T=60$ (similar results held at other sample sizes).
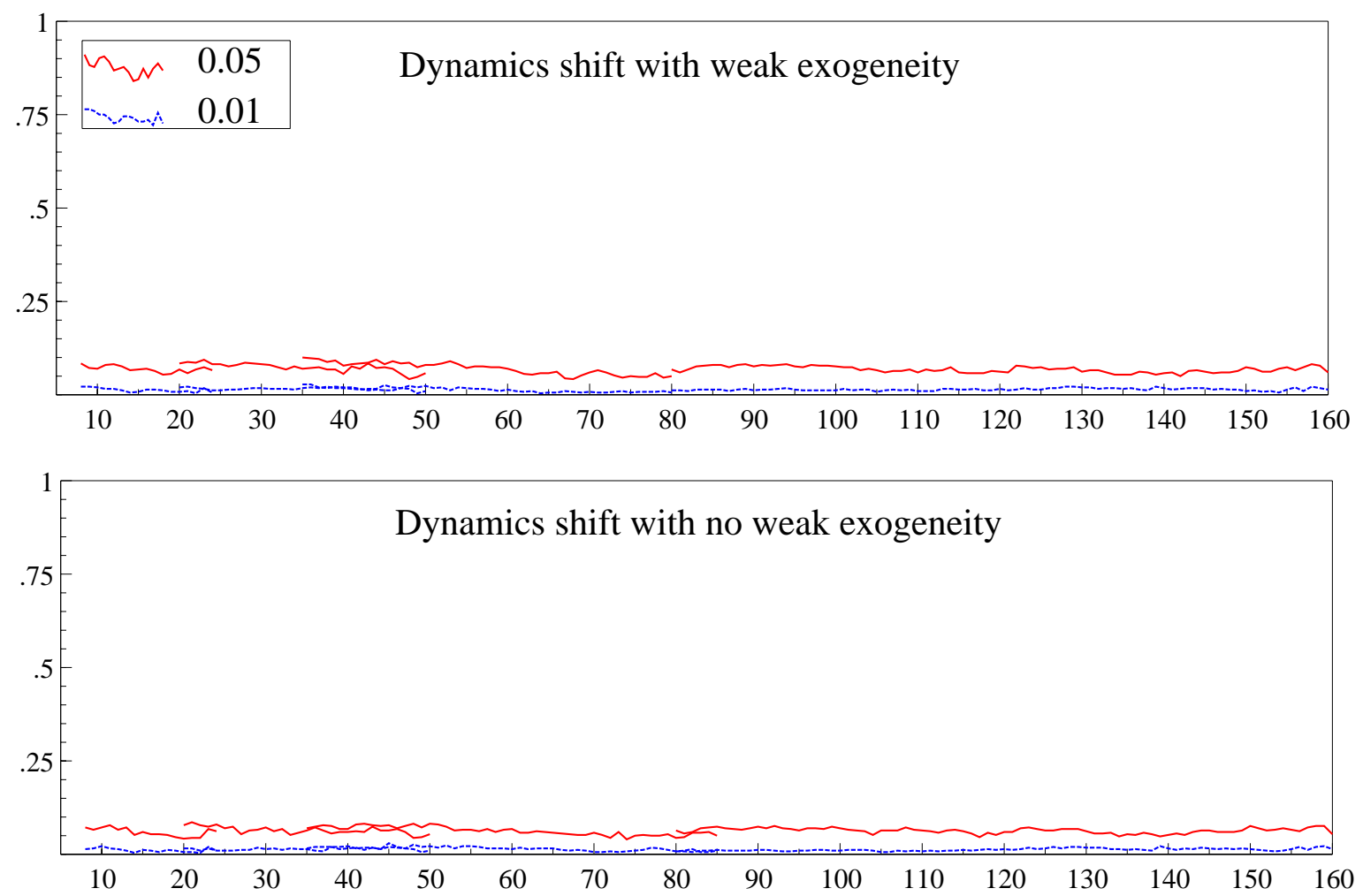

Figure 2 Constancy-test rejection frequencies for changes in $\alpha$. 
The remarkable feature of this set of experiments is that most of the parameters in the system have been changed, namely from:

$$
\boldsymbol{\Gamma}=\left(\begin{array}{cc}
0.9 & 0.1 \\
0.1 & 0.9
\end{array}\right) ; \boldsymbol{\tau}=\left(\begin{array}{r}
0.11 \\
-0.09
\end{array}\right)
$$

to:

$$
\boldsymbol{\Gamma}^{*}=\left(\begin{array}{cc}
0.85 & 0.15 \\
0.1 & 0.9
\end{array}\right) ; \quad \boldsymbol{\tau}^{*}=\left(\begin{array}{r}
0.16 \\
-0.09
\end{array}\right),
$$

yet the data hardly alter. Moreover, increasing the size of the shift in $\alpha_{1}$, and indeed making both $\alpha$ s shift, does not improve the detectability: for example, using $\alpha_{1}^{*}=-0.2$, and $\alpha_{2}^{*}=-.15$ causes no perceptible increase in the rejection frequency, or movement in the recursive estimates, over those shown in figs. 2 and 3.
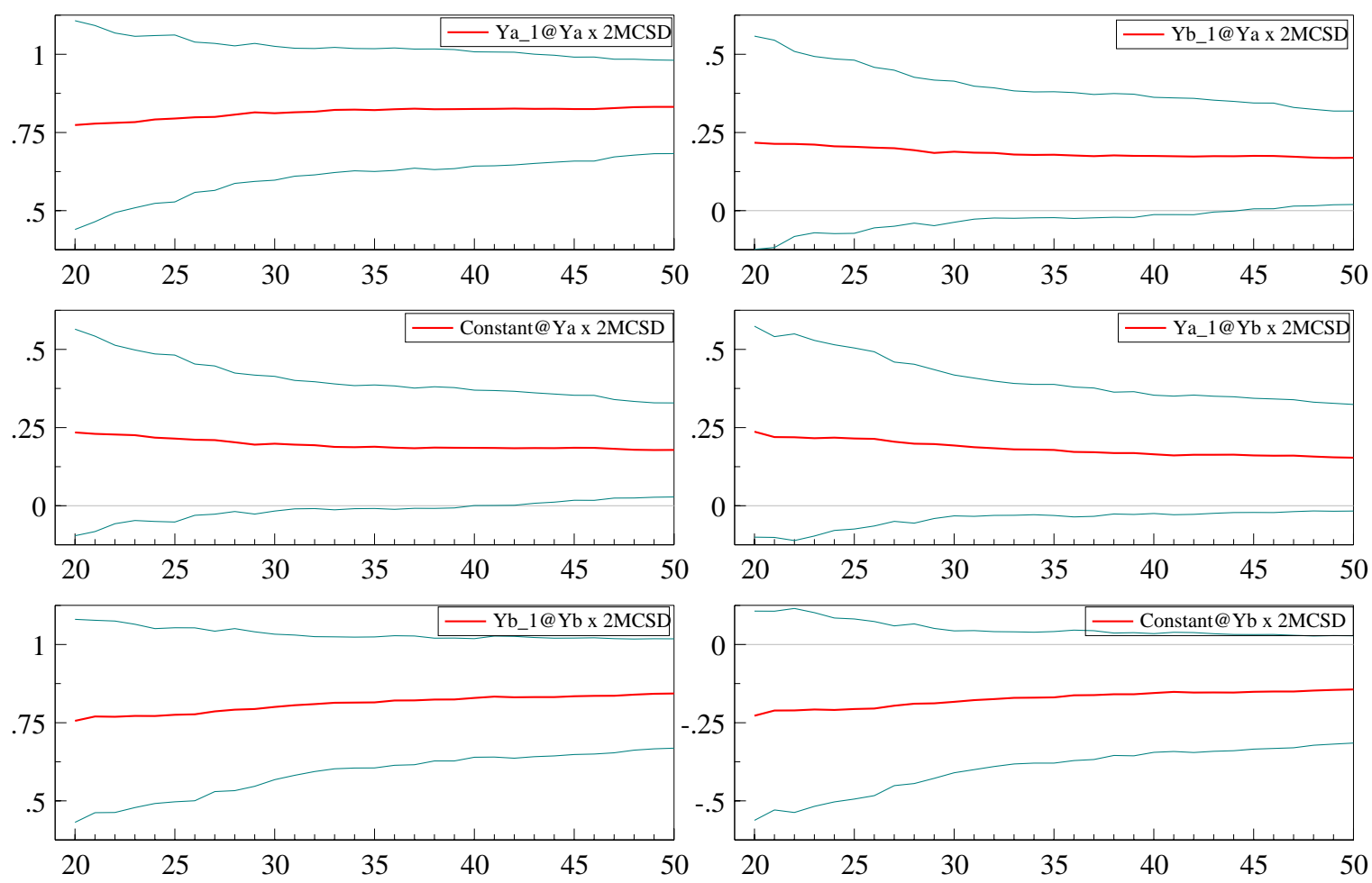

Figure 3 Recursively computed parameter estimates with $\pm 2 \hat{\sigma}$ at $T=60$.

The detectability of a shift in dynamics is dependent on whether the $\alpha$ s are increased or decreased: for example, setting $\alpha_{1}^{*}=\alpha_{2}^{*}=0$, so that cointegration vanishes and the DGP becomes a VAR in first differences, delivers the graphs in fig. 4 . The powers are a little better: but the highest power is still less than $30 \%$ even though in some sense, the vanishing of cointegration might be deemed a major structural change to an economy. The re-instatement of cointegration is somewhat more detectable than its loss, despite the earlier break not being modelled: intuitively, after a period without cointegration, the $x$ s have drifted apart, so the re-imposition has a marked deterministic effect, whereas when cointegration has operated for a time, the cointegration vector will be near its equilibrium mean, and hence switching it off will have only a small deterministic effect. 

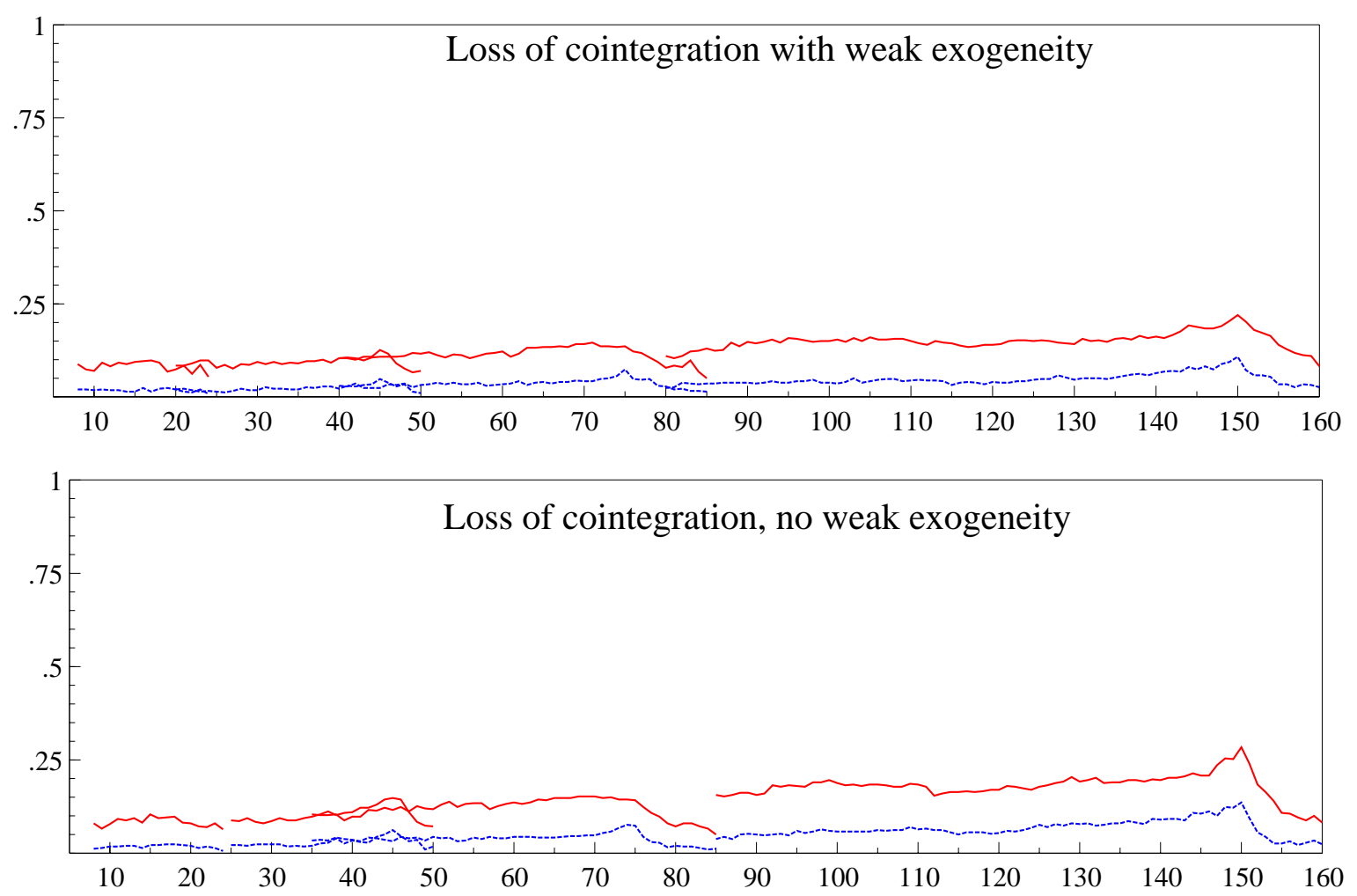

Figure 4 Constancy-test rejection frequencies for loss of cointegration.

\subsection{Equilibrium-mean shift}

Experiments in (C) show the contrasting ease of detecting equilibrium-mean breaks. Figure 5 confirms the anticipated outcomes for the mean shifts: the break-point test rejection frequencies are close to their nominal size of $5 \%$ under the null; the break in the equilibrium mean is easily detected, even at quite small sample sizes, especially when the EqCM enters both relations, which also serves to sharpen the location of the break. Because the relative positions of the breaks are held fixed, the power only increase slowly at $p=0.05$ as $T$ grows, but has a more marked effect on $p=0.01$ : larger samples do not ensure higher power for detecting the breaks.

To emphasize the detectability issue, note that the corresponding VAR here has the same $\Gamma$ as (16) throughout, and:

$$
\boldsymbol{\tau}=\left(\begin{array}{r}
0.11 \\
-0.09
\end{array}\right) \text { changes to } \boldsymbol{\tau}^{*}=\left(\begin{array}{r}
0.14 \\
-0.12
\end{array}\right) .
$$

Without the underlying theory to explain this outcome, it might seem astonishing that (18) can cause massive forecast failure, yet a shift from (16) to:

$$
\boldsymbol{\Gamma}^{*}=\left(\begin{array}{cc}
0.80 & 0.20 \\
0.15 & 0.85
\end{array}\right) ; \quad \boldsymbol{\tau}^{*}=\left(\begin{array}{r}
0.21 \\
-0.14
\end{array}\right)
$$

is almost completely undetectable.

\subsection{Growth-rate shift}

Finally, experiments in (D) examine the ease of detecting the corresponding doubling of the growth rate. This is a large change in real growth, but that is equivalent to a fraction of the change in (C), and at about $5 \%$ rejection, is again almost undetectable on these tests at small $T$, but now does become 


\section{Equilibrium-mean shift with weak exogeneity}
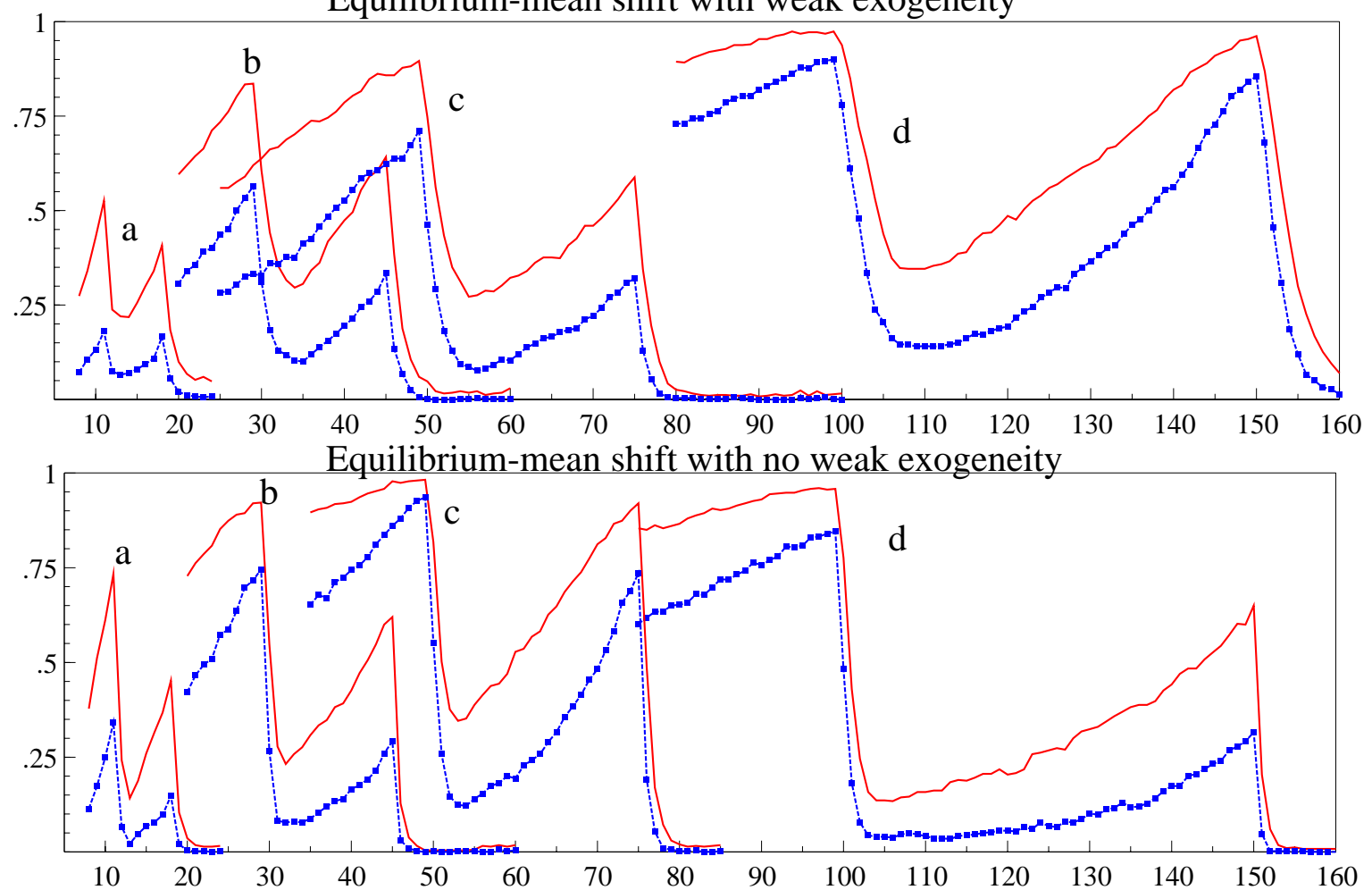

Figure 5 Constancy-test rejection frequencies for changes in $\boldsymbol{\mu}$.

increasingly easy to spot as the sample size grows. This is sensible, since the data exhibit a broken trend, but the model does not, so larger samples with the same relative break points induce effects of a larger magnitude. Thus, the type of structural break to be detected affects whether larger samples will help.
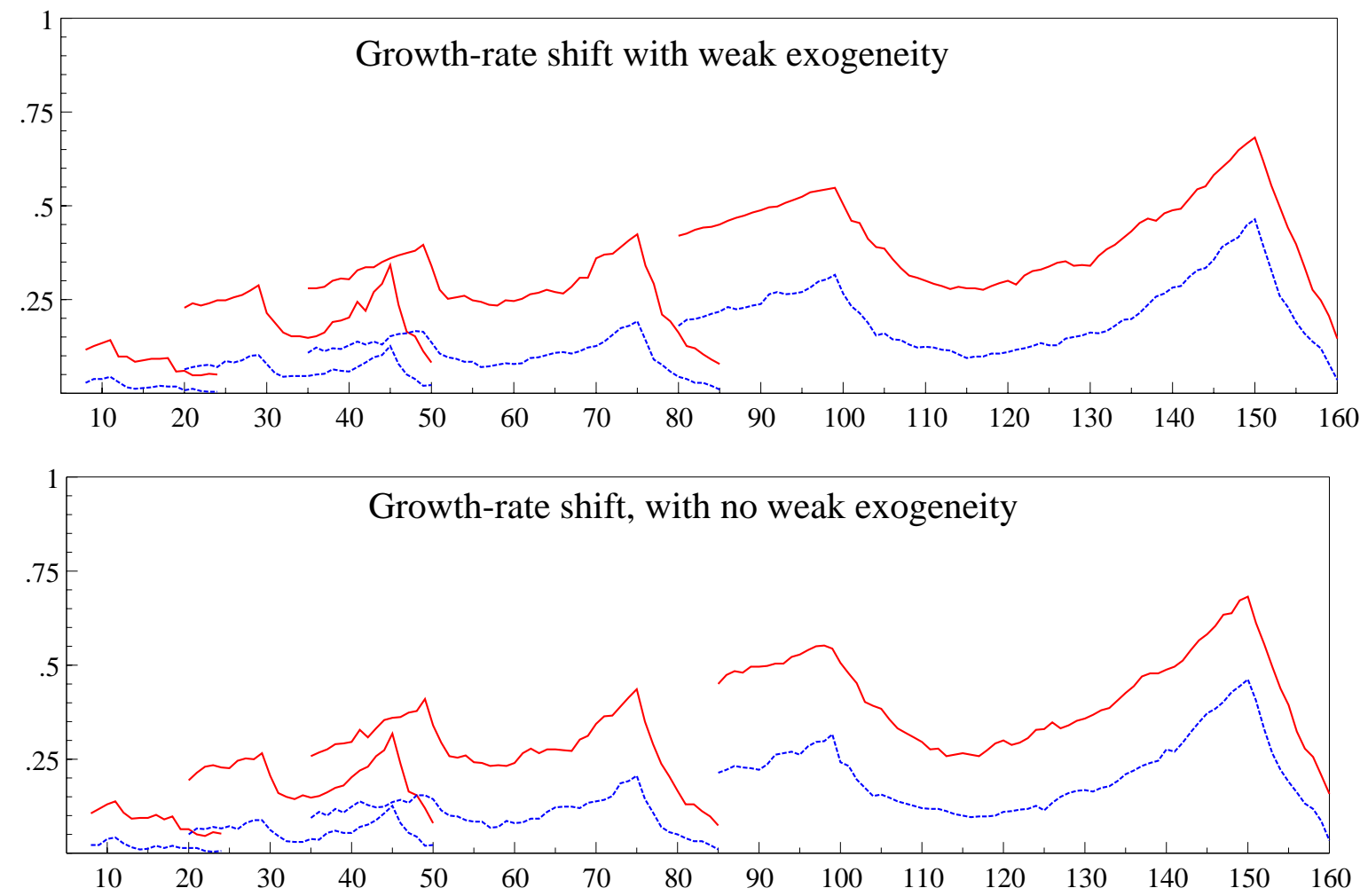

Figure 6 Constancy-test rejection frequencies for changes in $\gamma$. 
The model formulation also matters: if a VEqCM is used, the first growth break shows up much more strongly, as fig. 7 illustrates. The upper panel shows the break-point test rejection frequencies for the same experiment as fig. 6; the lower panel records the recursively-computed growth coefficients in the first equation with $\pm 2 S E$. This increased initial detectability is because the VEqCM 'isolates' the shift in $\gamma$, whereas the VAR 'bundles' it with $\gamma-\boldsymbol{\alpha} \boldsymbol{\mu}$, where the second component can be relatively much larger, camouflaging the shift. Moreover, VAR estimates of $\boldsymbol{\gamma}-\boldsymbol{\alpha} \boldsymbol{\mu}$ generally have very large standard errors because of the I(1) representation, whereas estimates of $\gamma$ are usually quite precise. Even so, a large growth-rate change has a surprisingly small effect at $T=200$ on the recursively-estimated intercept.
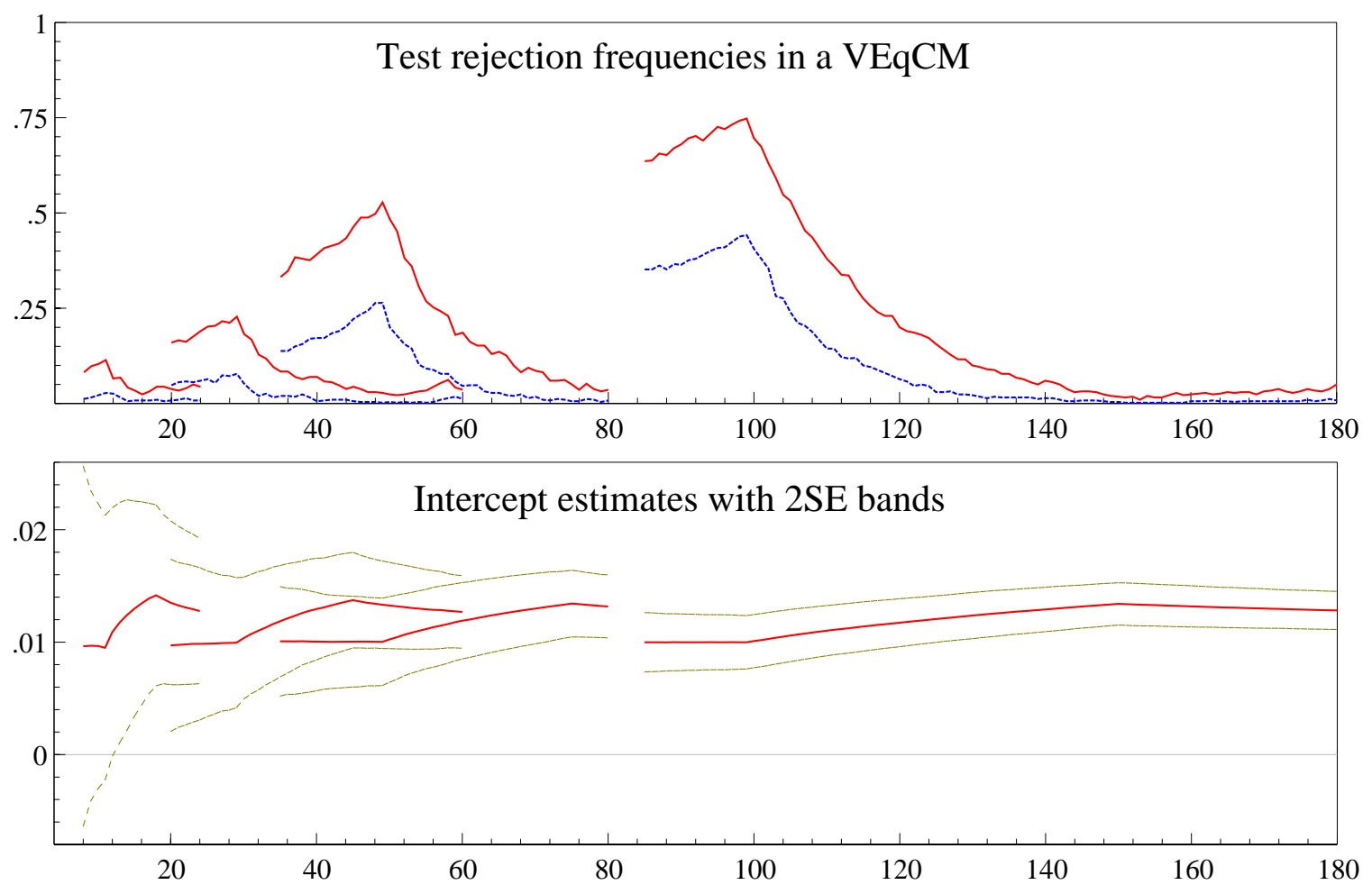

Figure 7 Constancy-test rejection frequencies in VEqCM and intercept estimates.

\subsection{Cointegration changes}

The main difficulty in considering changes in $\boldsymbol{\beta}$ is to ensure that these actually occur, and are not just linear recombinations, potentially offset by changes to $\alpha$ so $\boldsymbol{\Gamma}$ is unaltered. At the same time, one must isolate their impact from induced effects, including induced rank changes, and changes to $\boldsymbol{\mu}$. The first problem is due to transformations from the class of non-singular matrices $\mathbf{H}$ such that $\boldsymbol{\alpha}^{*}\left(\boldsymbol{\beta}^{*}\right)^{\prime}=\boldsymbol{\alpha} \mathbf{H H}^{-1} \boldsymbol{\beta}^{\prime}=\boldsymbol{\alpha} \boldsymbol{\beta}^{\prime}$ under which $\boldsymbol{\Gamma}$ is invariant. We have considered the second of these indirectly above when $\alpha$ changed from a non-zero value to zero, then back. The third requires that $\mathrm{E}\left[\left(\boldsymbol{\beta}^{*}\right)^{\prime} \mathbf{x}_{t}\right]=\boldsymbol{\mu}^{*}$ be known numerically in the Monte Carlo so that the disequilibrium remains at mean zero. In practice, changes in cointegration parameters almost certainly induce changes in equilibrium means, so will be detectable via the latter at a minimum.

The experiment to illustrate this case therefore set $\boldsymbol{\mu}=\boldsymbol{\mu}^{*}=\mathbf{0}$, with the other design parameters as before, and changed $\boldsymbol{\beta}^{\prime}$ from $(1:-1)$ to $(1:-0.9)$, altering $\boldsymbol{\tau}=\boldsymbol{\gamma}$ to ensure $\boldsymbol{\beta}^{\prime} \boldsymbol{\gamma}=\mathbf{0}$ both before and after the shift, and commencing the simulation from $\mathbf{y}_{0}^{\prime}=(0: 0)$, but discarding the first 20 generated observations. Since the process drifts, power should rise quickly as the sample size grows, both because 
of increased evidence, and the increased data values. Moreover, the re-instatement should be more detectable than the initial change, matching when cointegration is first lost then re-appears. Conversely, the induced change to $\boldsymbol{\Gamma}$ is not very large when $\boldsymbol{\alpha}^{\prime}=(-0.1: 0)$ :

$$
\boldsymbol{\Gamma}=\left(\begin{array}{cc}
0.9 & 0.1 \\
0.0 & 1.0
\end{array}\right) ; \text { whereas } \boldsymbol{\Gamma}^{*}=\left(\begin{array}{cc}
0.9 & 0.09 \\
0.0 & 1.0
\end{array}\right)
$$

with appropriate changes to $\tau$. In one sense, the very small shift in $\boldsymbol{\Gamma}$ is remarkably detectable, and reveals how important a role is played by shifts in off-diagonal elements in a VAR: we note with interest that the so-called 'Minnesota' prior shrinks precisely these terms towards zero (see Doan, Litterman and Sims, 1984). Equally, a slightly bigger change results when $\boldsymbol{\alpha}^{\prime}=(-0.1: 0.1)$, as:

$$
\boldsymbol{\Gamma}=\left(\begin{array}{cc}
0.9 & 0.1 \\
0.1 & 0.9
\end{array}\right) ; \text { whereas } \boldsymbol{\Gamma}^{*}=\left(\begin{array}{cc}
0.9 & 0.09 \\
0.1 & 0.91
\end{array}\right)
$$

and correspondingly, the power is higher in larger samples: see fig. 8 .
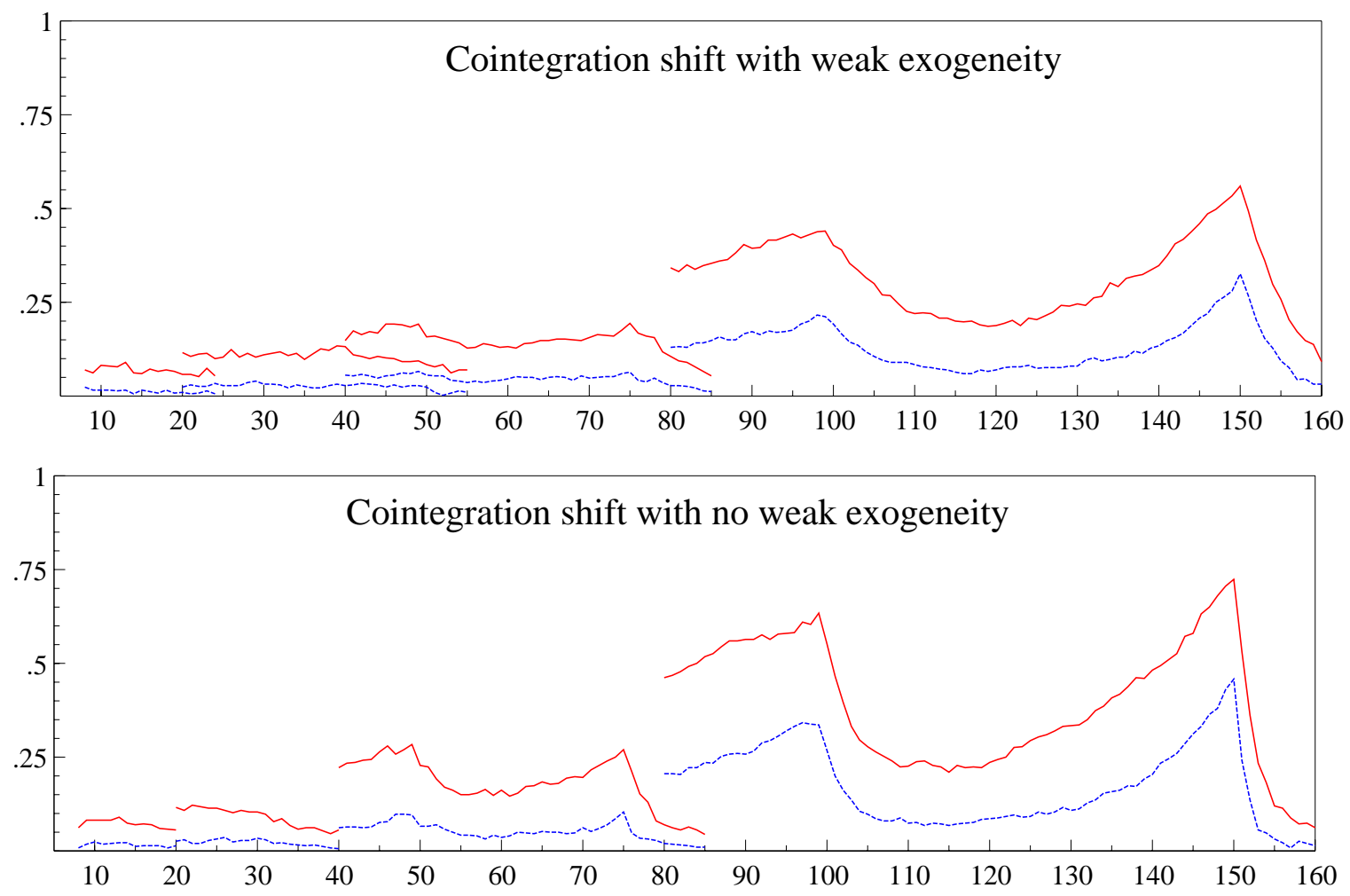

Figure 8 Rejection frequencies for a change in the cointegration parameter.

\section{Potential explanations}

Based on the taxonomy of possible sources of forecast errors in Clements and Hendry (1994), both Hendry and Doornik (1997) and Clements and Hendry (1998) link the non-detectability to the mean-zero nature of the variables whose parameters are changed. In their taxonomy, some forecasting mistakes derive from parameter changes which multiply the values of the regressors at the forecast origin: when such values are precisely zero, no errors can eventuate. Conversely, other changes multiply the data means (for $\mathrm{I}(0)$ components at least) and these only vanish in certain combinations. This suggests that 
the former, and the appropriate annihilating combinations of the latter, will be hard to detect, and that mean changes will be easy. This matches our simulation results.

Next, Hendry and Doornik (1997) prove that parameter-shift combinations which leave equilibrium means unaltered are isomorphic to mean-zero effects. They illustrate this result by an I(0) example akin to case (B) above, where the intercepts and dynamics are changed, but the equilibrium mean is constant, and show that the detectability is low. Clements and Hendry (1998) also demonstrate nearly equal detectability from inducing a shift of a given magnitude in the equilibrium mean by either an intercept shift, or by changing the coefficient of a regressor with a non-zero mean, even though the latter involves two sources of change (mean and reaction coefficient) and the former only one. This confirms that changes to reaction coefficients are primarily detectable through the induced mean shift.

A
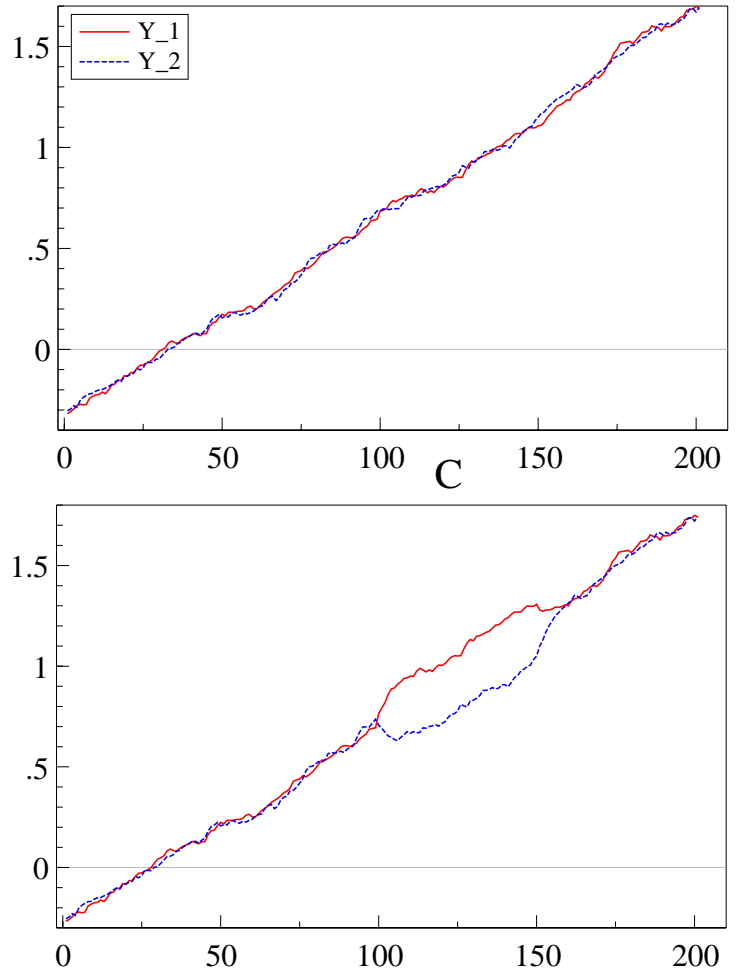

B
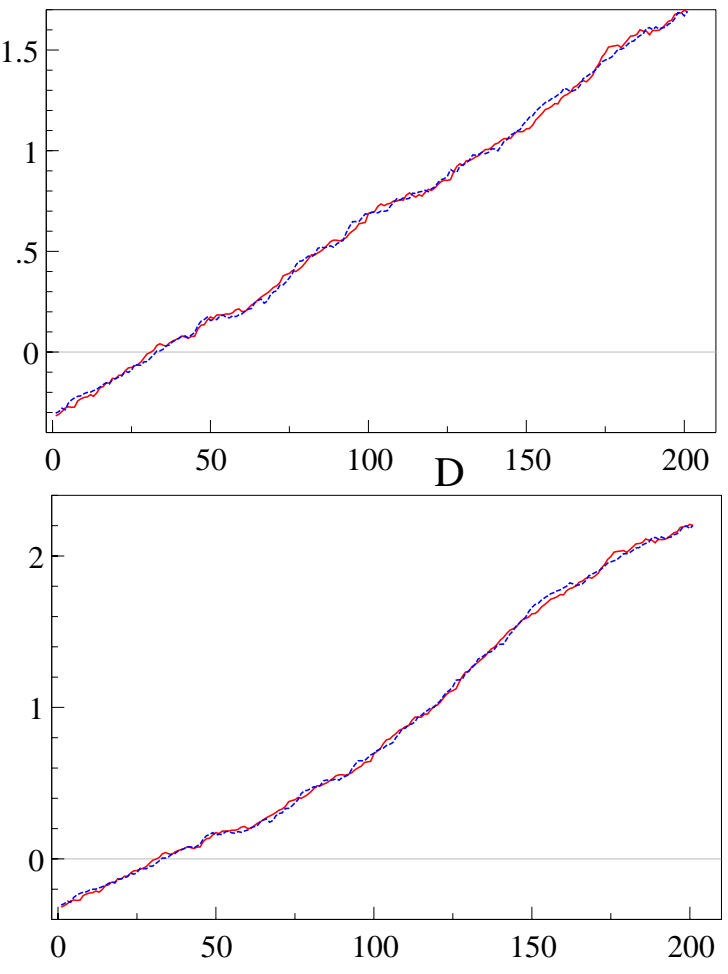

Figure 9 Data generated with and without structural breaks.

Indeed, the data generated by the constant DGP, and that suffering the shifts shown in (19), are almost the same, as fig. 9 (panels a, c) shows, whereas those from (16) or (D) are noticeably different (panels b, d respectively): both $\alpha$ s are non-zero in every panel. Such a feature is close to a lack of empirical identification, namely the evidence does not discriminate between two very different parameterizations, subject to maintaining the same means, and in fact, similar variances. Changing $\boldsymbol{\alpha}$ only induces variance shifts, and from the data graphs, these are seen to be small relative to the background 'noise'. Clements and Hendry (1999) derive the variance impacts of changes to $\boldsymbol{\alpha}$ which depend on $\nabla \boldsymbol{\alpha} \mathbf{V}_{\beta}(\boldsymbol{\nabla} \boldsymbol{\alpha})^{\prime}$ where $\mathbf{V}_{\beta}$ is the variance matrix of the cointegrating relations, $\left(\boldsymbol{\beta}^{\prime} \mathbf{x}_{t-1}-\boldsymbol{\mu}\right)$. When $\mathbf{V}_{\beta}$ is small, the data resulting from the shift will be only slightly different from the constant DGP, as fig. 9 shows, despite the substantive change in dynamics. Conversely, the deterministic shift is all too obvious.

Even bigger shifts in initially larger values of $\boldsymbol{\alpha}$ are still not easily detected. For the case:

$$
\boldsymbol{\Gamma}=\left(\begin{array}{ll}
0.7 & 0.3 \\
0.3 & 0.7
\end{array}\right) \text { to } \boldsymbol{\Gamma}^{*}=\left(\begin{array}{cc}
0.8 & 0.2 \\
0.15 & 0.85
\end{array}\right),
$$


A
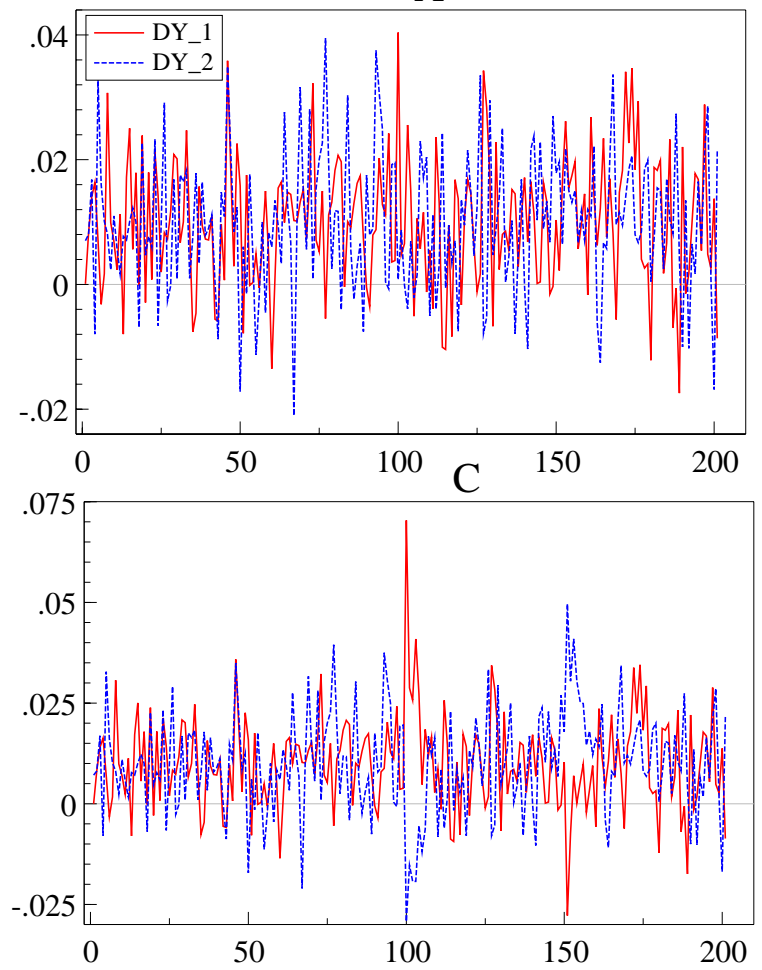

$\mathrm{B}$
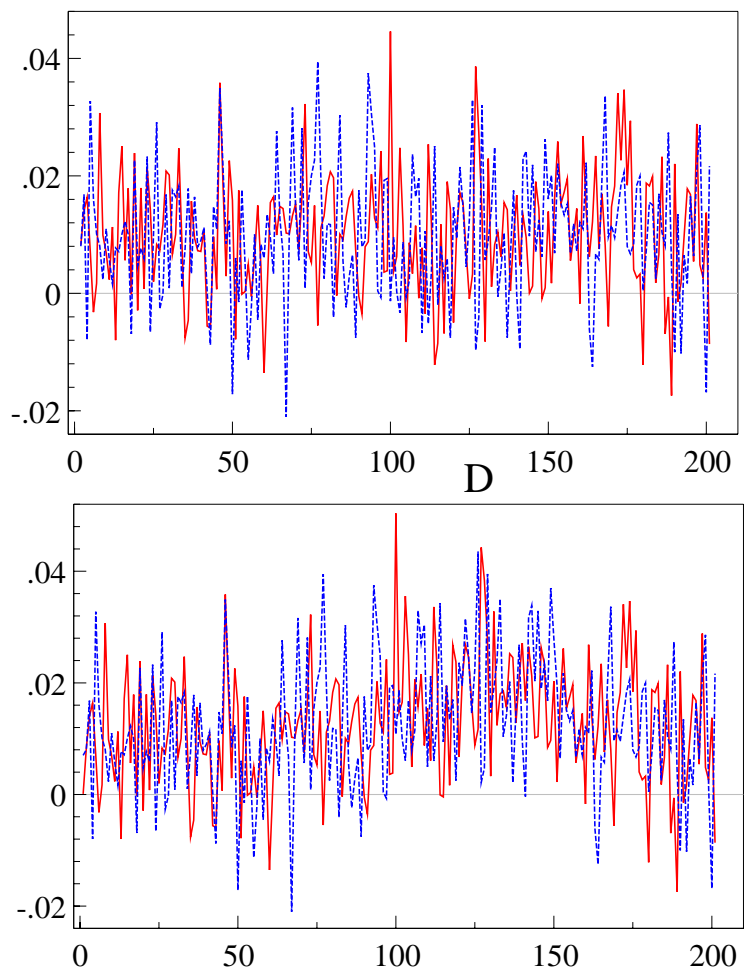

Figure 10 Differenced data generated with and without structural breaks.

$$
\boldsymbol{\tau}=\left(\begin{array}{r}
0.31 \\
-0.29
\end{array}\right) \text { to } \boldsymbol{\tau}^{*}=\left(\begin{array}{r}
0.21 \\
-0.14
\end{array}\right)
$$

the rejection-frequency outcomes are shown in fig. 11, with the corresponding data for the four sample sizes shown in fig. 12: the break points are far from obvious in the data, and the rejection frequencies remain low, and little improved by the increased sample size.

Finally, the algebra of the intercept in (3) also helps account for the outcomes in (D). There are two components to $\tau$ in a VAR, namely the unconditional growth rate, $\gamma$, and the equilibrium mean times the feedback, $\boldsymbol{\alpha} \boldsymbol{\mu}$. The former is almost inevitably small, whereas the latter can be huge, simply depending on the units of measurement, as discussed above. Often, therefore, $\widehat{\tau}$ is a large number, with a large standard error, suggesting its value is very uncertain, and hence - but be careful - that it can be changed considerably without much impact on goodness of fit. Certainly, small changes in $\widehat{\Gamma}$ can offset those in $\widehat{\tau}$ when estimating the VAR unrestrictedly, which is what the large standard errors reflect. However, consider estimating the VAR in (1) to 'calibrate' a Monte Carlo: given $\widehat{\Gamma}$ (to three digits say), round $\widehat{\tau}$ to three digits as well when the numbers are of the order of (say) 10.54 (SE $=2.1$ ), thereby using 10.5. The result will generate data wholly unlike those from which the VAR was estimated, because the implicit $\widehat{\gamma}$ has been altered from (say) 0.01 to -0.03 : instead of growing at about $4 \%$ p.a., the economy is vanishing at $12 \%$ p.a. ${ }^{2}$ Equally, orthogonalizing the equilibrium mean and the growth rate - a linear transform within the estimated model, to which it is invariant - can induce a dramatic change in the remaining intercept standard error, to (e.g.) 0.01 ( $\mathrm{SE}=0.004$ ). This helps explain the difficulty of detecting (D), and suggests that growth-rate changes will be far easier to detect in VEqCMs than VARs. Conversely, the VAR is more robust to such shifts from the perspective of forecast failure.

Finally, we have already explained why relatively tiny VAR parameter changes are readily detected when they correspond to shifts in cointegrating vectors.

\footnotetext{
${ }^{2}$ This occurred in early versions of the Monte Carlo DGP used in Doornik, Hendry and Nielsen (1998).
} 


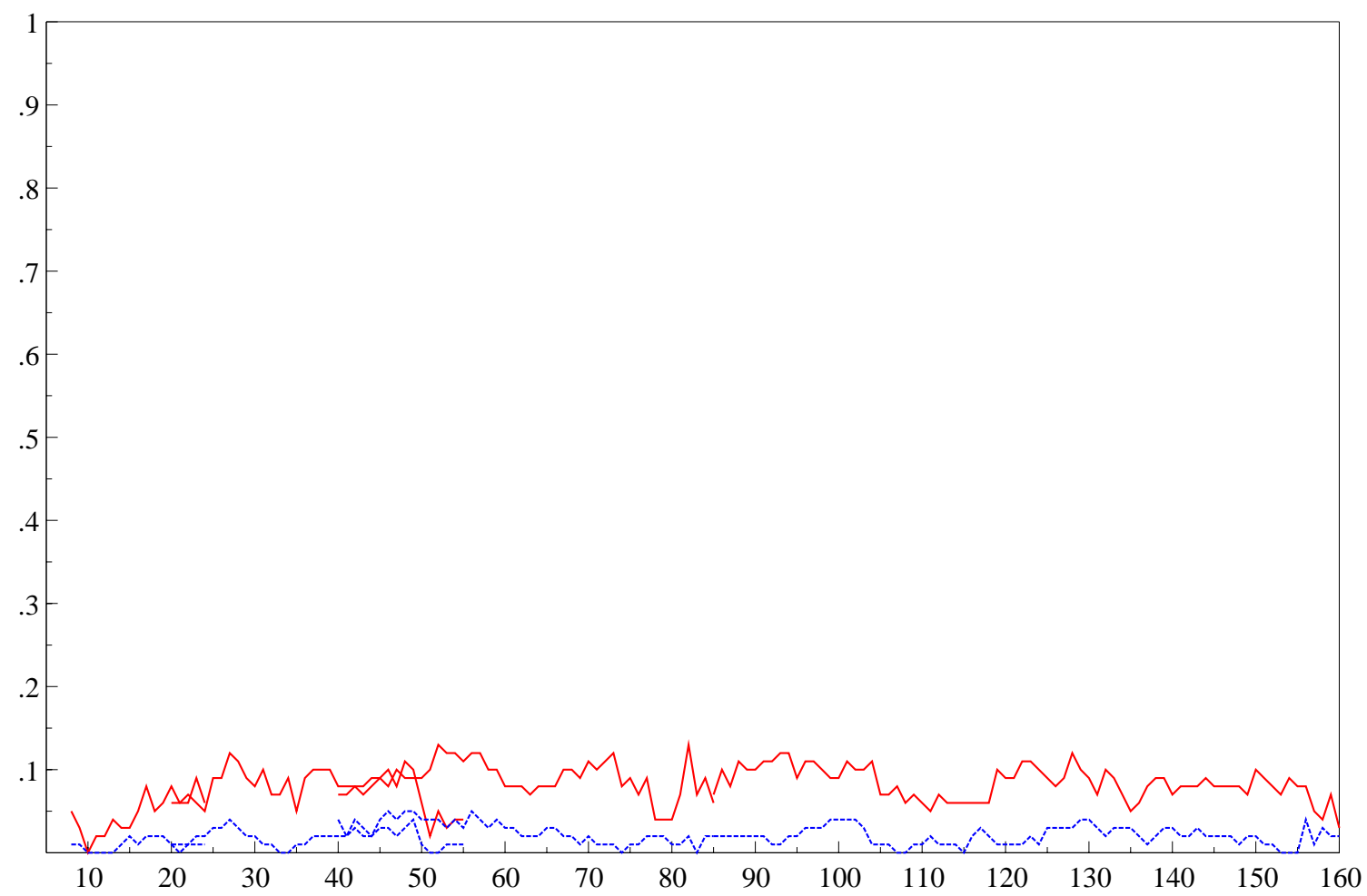

Figure 11 Rejection frequency for a large $\boldsymbol{\alpha}$ shift.
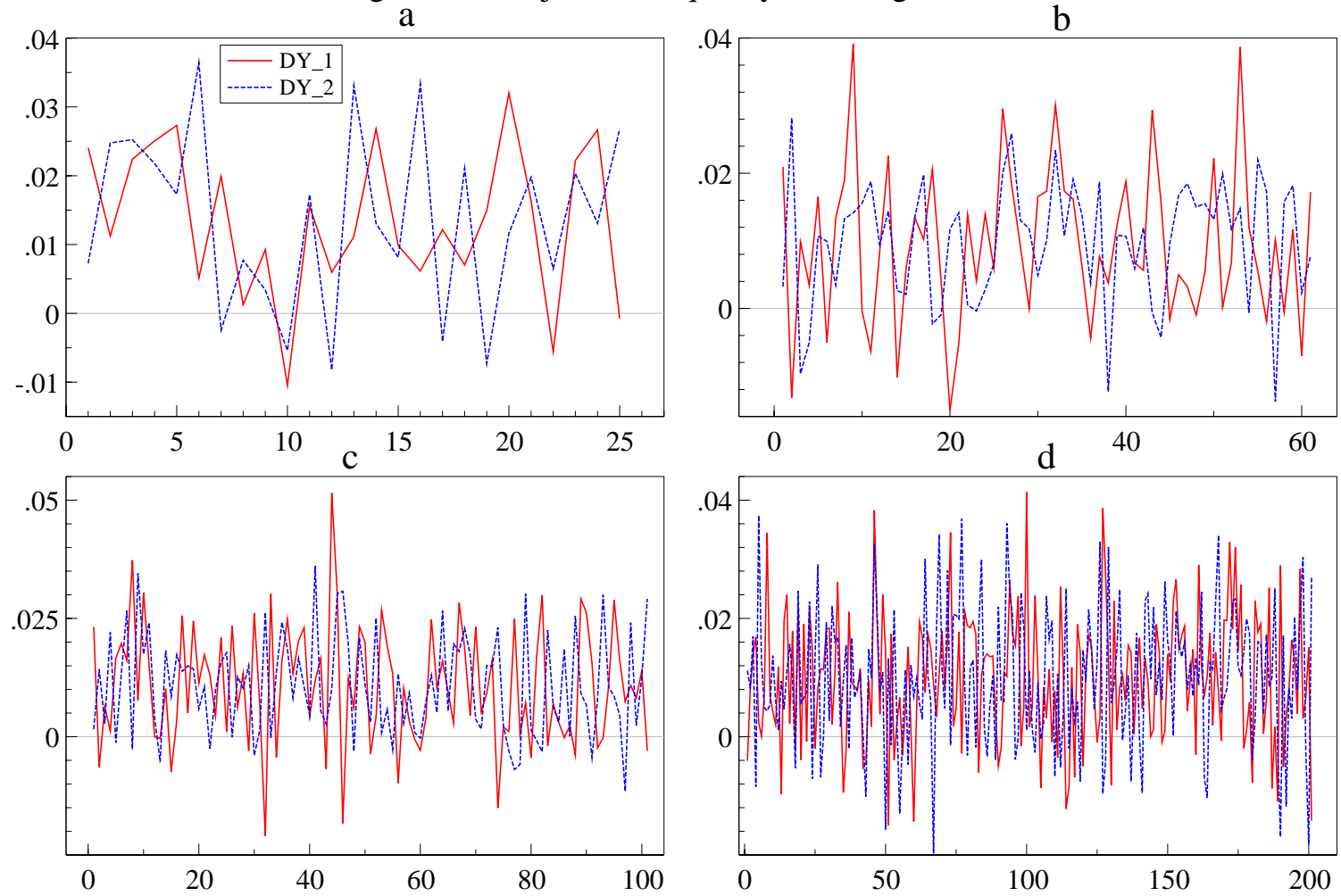

Figure 12 The differenced-data generated with a large dynamic break.

\section{Impulse-response analyses}

Such findings are potentially disastrous for 'impulse-response' analyses of economic policy. Since the changes in VAR intercepts and dynamic coefficient matrices may not be detected even when tested for, 
but the recorded estimates are a weighted average across the different regimes, the resulting impulse responses need not represent the policy outcomes that will in fact occur.

A Monte Carlo simulation, similar to those above, illustrates the problem, using the unrestricted I(0) VAR:

$$
\begin{aligned}
& y_{1, t}=\lambda_{1}+\phi_{11} y_{1, t-1}+\phi_{12} y_{2, t-1}+\epsilon_{1, t} \\
& y_{2, t}=\lambda_{2}+\phi_{21} y_{1, t-1}+\phi_{22} y_{2, t-1}+\epsilon_{2, t}
\end{aligned}
$$

where $\epsilon_{i, t} \sim \mathrm{IN}\left[0, \sigma_{i i}\right]$, with $\mathrm{E}\left[\epsilon_{1, t} \epsilon_{2, s}\right]=0 \forall t, s$. The baseline dynamic parameter values are $\phi_{11}=$ $0.50, \phi_{12}=\phi_{21}=-0.20$ and $\phi_{22}=-0.25$. We consider breaks in the $\phi_{i j}$ with constant unconditional expectations of zero. The full-sample size is $T=120$, with a single break at $t=0.5 T$. The unrestricted VAR with intercept and one lag is estimated, and then tested for breaks. The critical values for the constancy tests are those for a known break point, which delivers the highest possible power for the test used.

The rejection frequencies are reported graphically for both $p$ values: 1000 replications are used, and rejection frequencies at both 0.05 and 0.01 nominal test sizes are recorded (standard errors about 0.007 and 0.003 respectively). The graphs serve to illustrate the outcomes visually, showing that rejection frequencies are everywhere low in most cases, but confirming that the highest power is immediately before the break.

\subsection{Test size}

As fig. 13 reveals, the null rejection frequencies in the $\mathrm{I}(0)$ baseline data are reassuring: with 1000 replications, the approximate $95 \%$ confidence intervals are $(0.036,0.064)$ and $(0.004,0.016)$ for $5 \%$ and $1 \%$ nominal, and these are shown on the graphs as dotted and dashed lines respectively. The actual test sizes are close to their nominal levels.

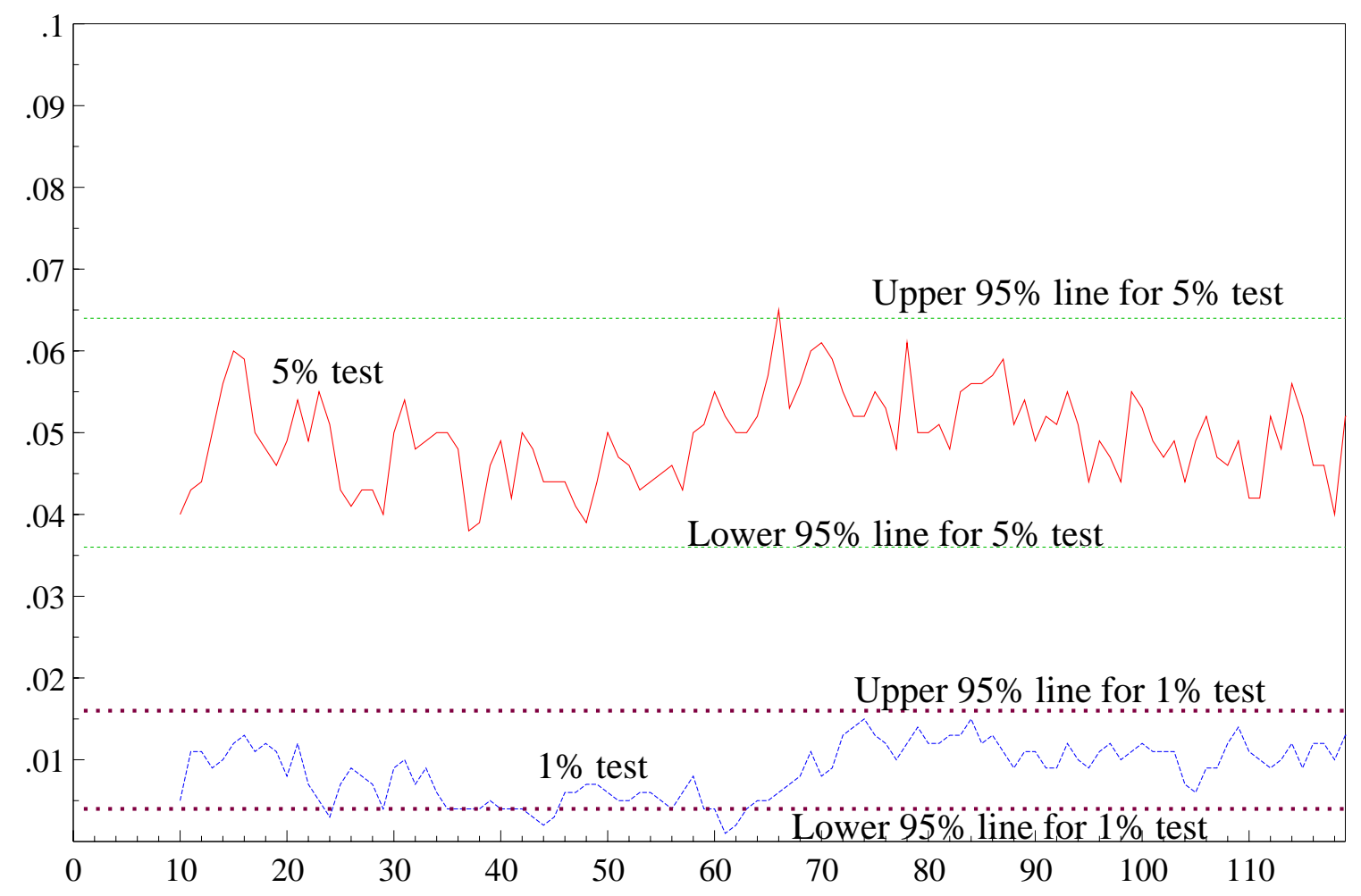

Figure 13 Constancy-test rejection frequencies for the $\mathrm{I}(0)$ null. 


\subsection{I(0) dynamic shift}

The detectability of a shift in dynamics is low when the DGP is an I(0) VAR with $\boldsymbol{\lambda}=\mathbf{0}$. We consider a large parameter shift, from:

$$
\mathbf{\Phi}=\left(\begin{array}{cc}
0.50 & -0.20 \\
-0.20 & -0.25
\end{array}\right) \text { to } \boldsymbol{\Phi}^{*}=\left(\begin{array}{cc}
0.50 & 0.20 \\
0.20 & 0.25
\end{array}\right)
$$

The first element is left constant to highlight the changes in the other impulses. This break delivers the graph in fig. 14. The highest power is less than $25 \%$, even though the change constitutes a major structural break for the model economy.

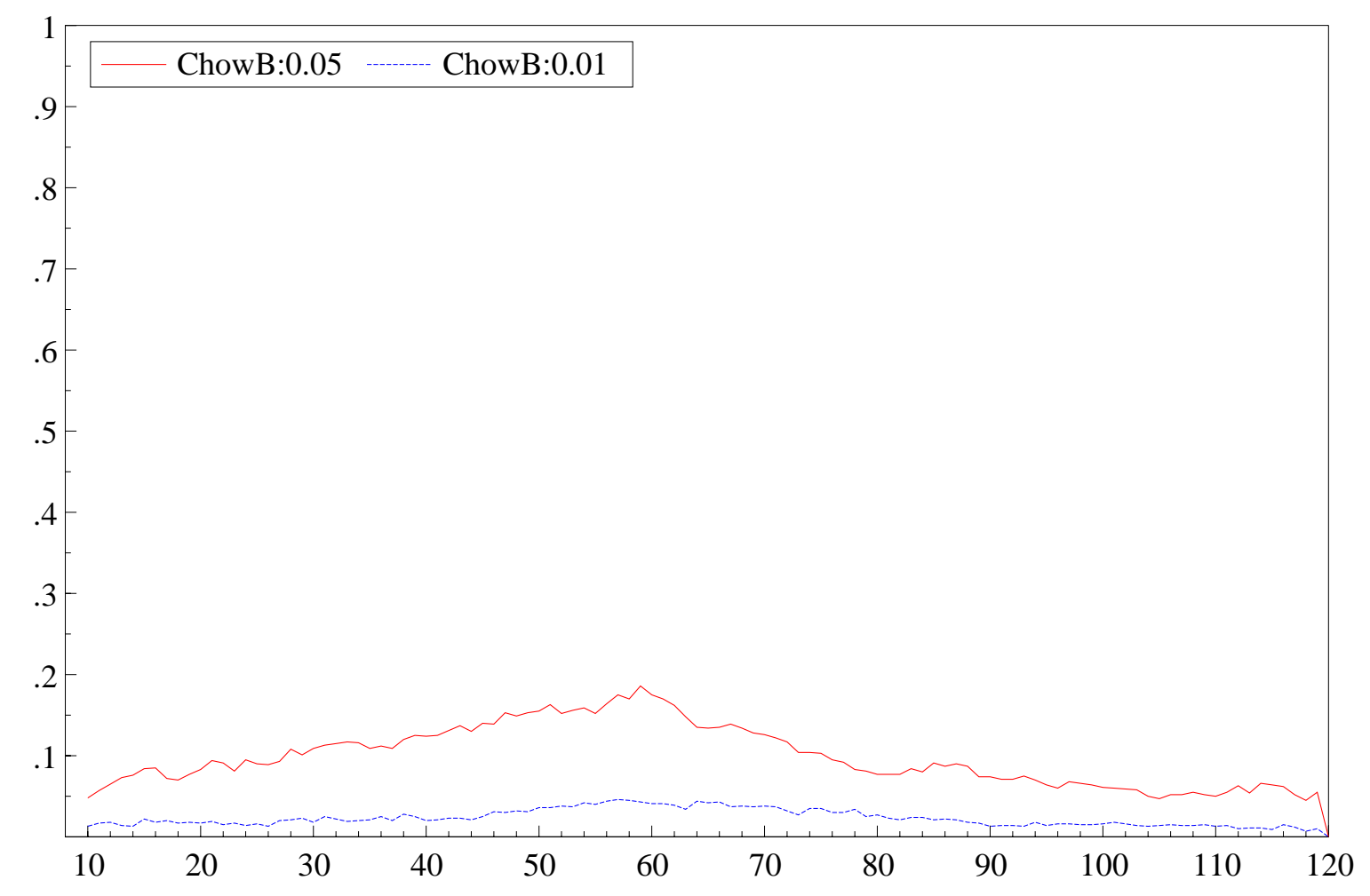

Figure 14 Constancy-test rejection frequencies for the $\mathrm{I}(0)$ structural break.

\subsection{Impulse responses}

Finally, we record the impulse responses from the pre- and post- break models, and the model fitted across the regime shifts in fig. 15. The contrast is marked: despite the near undetectability of the break, the signs of most of the impulses have altered, and those obtained from the fitted model sometimes reflect one regime, and sometimes the other. Overall, mis-leading policy advice would follow.

\section{Conclusion}

With I(1) data generated from a cointegrated VAR, the detectability of a change is not well reflected by the original VAR parameterization. Apparently-large shifts in both the VAR intercept and dynamic coefficient matrix need not be detectable, whereas seemingly tiny changes can have a substantial and easily detected, effect. Viewing the issue through a vector equilibrium-correction parameterization helps clarify this outcome. Moreover, it reveals that equilibrium-mean shifts are readily detectable, whereas 

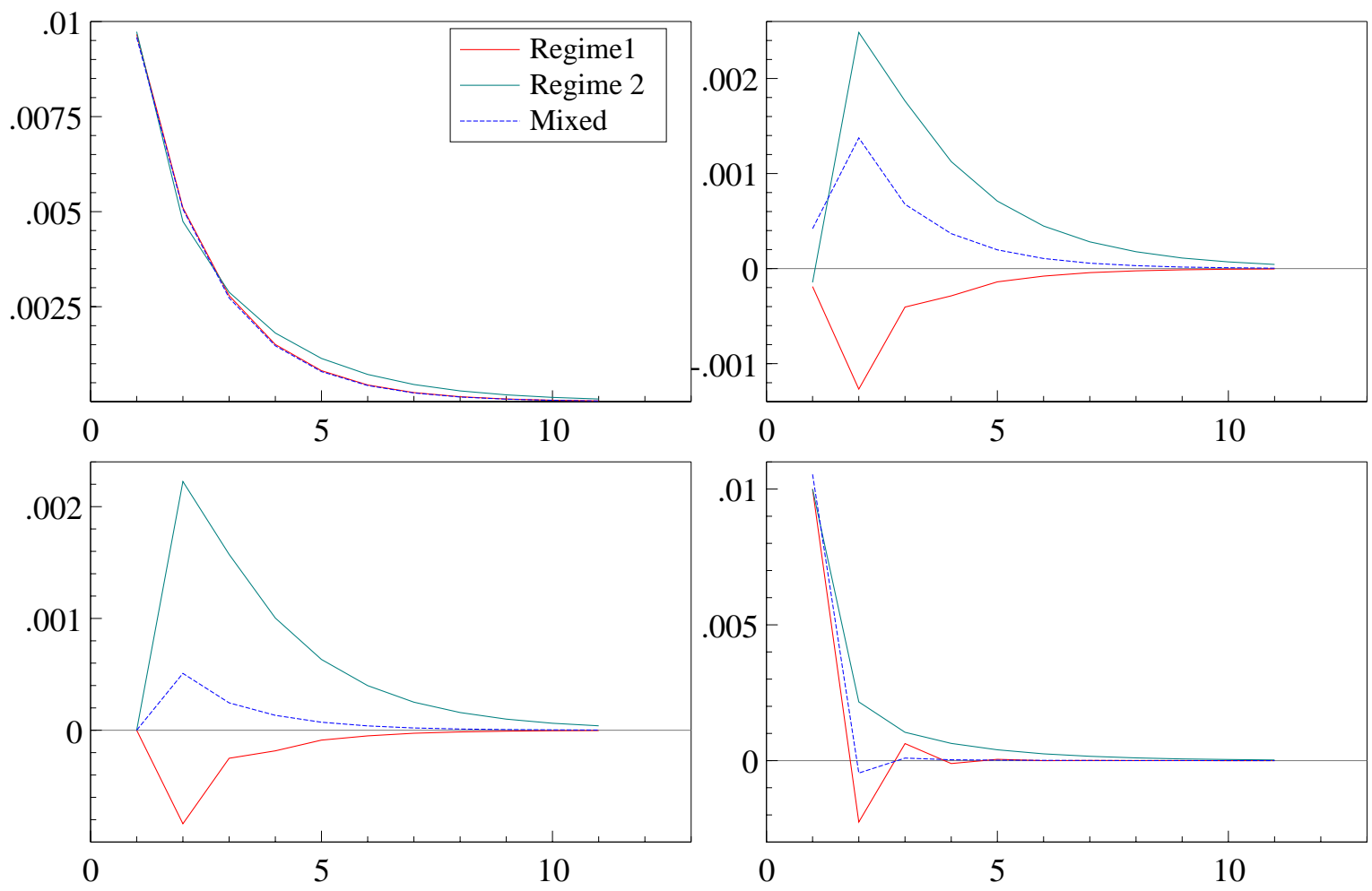

Figure 15 Impulse response comparisons in an I(0) VAR.

mean-zero shifts are not. Thus, the implicit variation-free assumptions about parameters are crucial in a world of structural shifts, with consequential benefits of robustness in forecasting versus drawbacks of non-detection in modelling. Other tests, including monitoring and variance-change tests, merit consideration, but overall the results suggest a focus on mean shifts to detect changes of concern to macro-economic forecasting.

Such findings are potentially disastrous for 'impulse-response' analyses of economic policy. Since the changes in VAR intercepts and dynamic coefficient matrices may not be detected even when tested for, but the recorded estimates are a weighted average across the different regimes, the resulting impulse responses do not represent the policy outcomes that will in fact occur.

\section{References}

Anderson, G. J., and Mizon, G. E. (1983). Parameter constancy tests: Old and new. Discussion Paper in Economics and Econometrics, no.8325, Economics Department, University of Southampton.

Anderson, G. J., Mizon, G. E., and O'Brien, R. J. (1993). Testing for parameter constancy in systems of $\mathrm{I}(0)$ variables. mimeo., European University Institute, Florence, Italy.

Anderson, T. W. (1984). An Introduction to Multivariate Statistical Analysis, 2nd edn. New York: John Wiley \& Sons.

Brown, R. L., Durbin, J., and Evans, J. M. (1975). Techniques for testing the constancy of regression relationships over time (with discussion). Journal of the Royal Statistical Society B, 37, 149-192.

Chow, G. C. (1960). Tests of equality between sets of coefficients in two linear regressions. Econometrica, 28, 591-605.

Clements, M. P., and Hendry, D. F. (1994). Towards a theory of economic forecasting. In Hargreaves, 
C. (ed.), Non-stationary Time-series Analysis and Cointegration, pp. 9-52. Oxford: Oxford University Press.

Clements, M. P., and Hendry, D. F. (1998). On winning forecasting competitions in economics. mimeo, Institute of Economics and Statistics, University of Oxford.

Clements, M. P., and Hendry, D. F. (1999). Forecasting Non-stationary Economic Time Series: The Zeuthen Lectures on Economic Forecasting. Cambridge, Mass.: MIT Press. Forthcoming.

Doan, T., Litterman, R., and Sims, C. A. (1984). Forecasting and conditional projection using realistic prior distributions. Econometric Reviews, 3, 1-100.

Doornik, J. A. (1996). Object-Oriented Matrix Programming using Ox. London: International Thomson Business Press and Oxford: http://www.nuff.ox.ac.uk/Users/Doornik/.

Doornik, J. A., and Hendry, D. F. (1997). Modelling Dynamic Systems using PcFiml 9 for Windows. London: Timberlake Consultants Press.

Doornik, J. A., and Hendry, D. F. (1998). Monte Carlo simulation using PcNaive for Windows. Unpublished typescript, Nuffield College, University of Oxford.

Doornik, J. A., Hendry, D. F., and Nielsen, B. (1998). Inference in cointegrated models: UK M1 revisited. Journal of Economic Surveys, 12, 533-572.

Engle, R. F., Hendry, D. F., and Richard, J.-F. (1983). Exogeneity. Econometrica, 51, 277-304. Reprinted in Hendry, D. F., Econometrics: Alchemy or Science? Oxford: Blackwell Publishers, 1993; and in Ericsson, N. R. and Irons, J. S. (eds.) Testing Exogeneity, Oxford: Oxford University Press, 1994.

Ericsson, N. R., Hendry, D. F., and Prestwich, K. M. (1998). The demand for broad money in the United Kingdom, 1878-1993. Scandinavian Journal of Economics, 100, 289-324.

Hendry, D. F. (1994). HUS revisited. Oxford Review of Economic Policy, 10, 86-106.

Hendry, D. F. (1997). The econometrics of macro-economic forecasting. Economic Journal, 107, 13301357.

Hendry, D. F., and Doornik, J. A. (1997). The implications for econometric modelling of forecast failure. Scottish Journal of Political Economy, 44, 437-461. Special Issue.

Hendry, D. F., and Ericsson, N. R. (1991). Modeling the demand for narrow money in the United Kingdom and the United States. European Economic Review, 35, 833-886.

Hylleberg, S., and Mizon, G. E. (1989). Cointegration and error correction mechanisms. Economic Journal, 99, 113-125. Supplement.

Hylleberg, S., Mizon, G. E., and O’Brien (1993). Testing for parameter constancy in systems of cointegrated variables. Mimeo, European University Institute, Florence.

Rao, C. R. (1952). Advanced Statistical Methods in Biometric Research. New York: John Wiley.

Rao, C. R. (1973). Linear Statistical Inference and its Applications, 2nd edn. New York: John Wiley \& Sons. 